\title{
RANDOM PERMUTATIONS WITH LOGARITHMIC CYCLE WEIGHTS
}

\author{
NICOLAS ROBLES AND DIRK ZEINDLER
}

\begin{abstract}
We consider random permutations on $\mathfrak{S}_{n}$ with logarithmic growing cycles weights and study the asymptotic behavior as the length $n$ tends to infinity. We show that the cycle count process converges to a vector of independent Poisson variables and also compute the total variation distance between both processes. Next, we prove a central limit theorem for the total number of cycles. Furthermore we establish a shape theorem and a functional central limit theorem for the Young diagrams associated to random permutations under this measure. We prove these results using tools from complex analysis and combinatorics. In particular we have to apply the method of singularity analysis to generating functions of the form $\exp \left((-\log (1-z))^{k+1}\right)$ with $k \geq 1$, which have not yet been studied in the literature.
\end{abstract}

\section{INTRODUCTION}

Let $\mathfrak{S}_{n}$ be the symmetric group of all permutations on elements $1, \ldots, n$. For any permutation $\sigma \in \mathfrak{S}_{n}$, denote by $C_{m}=C_{m}(\sigma)$ the cycle counts, that is, the number of cycles of length $m=1, \ldots, n$ in the cycle decomposition of $\sigma$; clearly

$$
C_{m} \geq 0 \quad(m \geq 1), \quad \sum_{m=1}^{n} m C_{m}=n .
$$

Here we study random permutations with respect to the following probability measure.

Definition 1.1. Let $\Theta=\left(\theta_{m}\right)_{m \geq 1}$ be given, with $\theta_{m} \geq 0$ for every $m \geq 1$. We define for $\sigma \in \mathfrak{S}_{n}$ the weighted measures on $\mathfrak{S}_{n}$ as

$$
\mathbb{P}_{\Theta}[\sigma]:=\frac{1}{h_{n} n !} \prod_{m=1}^{n} \theta_{m}^{C_{m}}
$$

with $h_{n}=h_{n}(\Theta)$ a normalization constant and $h_{0}:=1$.

This measure has received a lot of attention in recent years and has been studied by many authors. An overview can be found in [11]. Classical cases of $\mathbb{P}_{\Theta}$ are the uniform measure $\left(\theta_{m} \equiv 1\right)$ and the Ewens measure $\left(\theta_{m} \equiv \theta\right)$. The uniform measure is well studied and has a long history (see e.g. the first chapter of [1] for a detailed account with references). The Ewens measure originally appeared in population genetics, see [12], but has also various applications through its connection with Kingman's coalescent process, see [16].

The motivation to study the measure $\mathbb{P}_{\Theta}$ has its origins in mathematical physics. Explicitly, it occurred in the context of the Feynman-Kac representation of the dilute Bose gas and it has been proposed in connection with the study of the Bose-Einstein condensation (see e.g. [4] and

Date: March 18, 2019.

1991 Mathematics Subject Classification. 60F17, 60F05, 60C05, $40 \mathrm{E} 05$.

Key words and phrases. random permutations, cycle counts, total variation distance, total number of cycles, singularity analysis, limit shape, functional central limit theorem, Tauberian theorem. 
[11]). An important question in this context, which is also interesting on its own right, is the possible emergence of cycles with a cycle length with order of magnitude $n$ as $n \rightarrow \infty$. It is clear that the asymptotic behaviour of the measure $\mathbb{P}_{\Theta}$ as $n \rightarrow \infty$ strongly depends on the sequence $\Theta=\left(\theta_{m}\right)_{m \geq 1}$. In the current literature, only the cases $\theta_{m} \approx \vartheta$ and $\theta_{m} \sim m^{\gamma}$ with $\gamma>0$ are well studied. It is known that in the case $\theta_{m} \approx \vartheta$ there are cycles of order $n$ in the limit and that the longest cycles follow a Poisson-Dirichlet distribution, see [17, 21, 11, 4]. On the other hand, it was shown in [8, 11] that in the case $\theta_{m} \sim m^{\gamma}$ most cycles have a cycle length of order $n^{\frac{1}{1+\gamma}}$ and thus there are no cycles of order $n$ in the limit. Furthermore, it was established in [8] that the Young diagrams associated to random permutations converges in this situation to a limit shape and in [9] an asymptotic shape of the cycle length distribution was determined. In this paper, we are mainly interested in the cycle weights of the form

$$
\theta_{m}=\log ^{k} m \text { for } m \in \mathbb{N} \text { and some } k \in \mathbb{N} .
$$

However, we do not require for our argumentation that $\theta_{m}$ be of the form in (1.3). We only require that the generating function

$$
g_{\Theta}(t):=\sum_{m=1}^{\infty} \frac{\theta_{m}}{m} t^{m}
$$

to have some analytic properties. We will determine these properties in Lemma 2.4 for the case $\theta_{m}=\log ^{k} m$ and use them in Section 2.3 as a basis to specify our working assumptions on $g_{\Theta}(t)$. We will also give some brief comments on which cycle weights fulfill these properties. Weights of the form (1.3) have not been studied in the literature and our motivation to consider these weights is the following question. Are there any cycles of order $n$ in the limit if one is considering slowly growing cycles weights $\theta_{m}$ as $m \rightarrow \infty$ ? We show in this paper that the length of typical cycle under this measure has the order of magnitude $n / \log ^{k} n$ (see Theorem 3.6 and Theorem 4.1) and thus there are no cycles with lengths of order $n$. Also, we show the following. For each $b \in \mathbb{N}$ fixed, we have as $n \rightarrow \infty$

$$
\left(C_{1}, C_{2}, \ldots, C_{b}\right) \stackrel{d}{\rightarrow}\left(Y_{1}, \ldots, Y_{b}\right)
$$

with $Y_{1}, \cdots, Y_{b}$ independent Poisson distributed random variables with $\mathbb{E}\left[Y_{m}\right]=\frac{\theta_{m}}{m}$, see Theorem 3.1. Further, we compute the total variation distance between both processes and show that this is tending to 0 for $b=o\left(n^{c}\right)$ for some $c \in(0,1)$, see Theorem 5.1. Moreover, we prove a central limit theorem for the total number of cycles, see Theorem 3.2, and show that a typical permutation consists in average of $\frac{\log ^{k+1}(n)}{k+1}$ disjoint cycles. Finally, we establish in Section 4 a shape theorem and a functional central limit theorem for the Young diagrams associated to random permutations.

We prove these results using tools from complex analysis and combinatorics. For this, we have in particular to compute the asymptotic behaviour of

$$
\left[z^{n}\right]\left[\exp \left((-\log (1-z))^{k+1}\right)\right] \text { as } n \rightarrow \infty,
$$

where $\left[z^{n}\right][f(z)]$ denotes the $n$ 'th Taylor coefficient in the expansion of $f(z)$ about $z=0$. As far as we are aware, this has not yet been studied in the literature and we compute (1.6) with a modified version of the saddle point method, see Theorem 2.5 .

Notation: We use standard notation $\mathbb{Z}$ and $\mathbb{N}$ for the sets of integer and natural numbers, respectively, and also denote $\mathbb{N}_{0}:=\{m \in \mathbb{Z}: m \geq 0\}=\{0\} \cup \mathbb{N}$. Furthermore, we use 
interchangeably the notion $f_{n}=O\left(g_{n}\right)$ and $f_{n} \ll g_{n}$ if there exists a constant $C>0$ and $n_{0} \in \mathbb{N}$ such that $\left|f_{n}\right| \leq C\left|g_{n}\right|$ for all $n \geq n_{0}$.

\section{GENERATING FUnCTIONS AND ASYMPtOTIC THEOREMS}

We recall in Section 2.1 some basic facts about $\mathfrak{S}_{n}$ and generating functions. This includes Pólya's Enumeration Theorem, which is a useful tool to perform averages on the symmetric group. In Section 2.2, we determine some analytic properties of the generating functions occurring in this paper and establish a result, see Theorem 2.5, which enables us to compute the asymptotic behaviour of the expression in (1.6).

2.1. Generating functions. For a sequence of complex numbers $\left(a_{m}\right)_{m \geq 0}$, its (ordinary) generating function is defined as the formal power series

$$
g(t):=\sum_{m=0}^{\infty} a_{m} t^{m}
$$

As usual [14, $\S \mathrm{I} .1$, p. 19], we define the extraction symbol $\left[t^{m}\right] g(t):=a_{m}$, that is, as the coefficient of $t^{m}$ in the power series expansion 2.1) of $g(t)$.

The following simple lemma known as Pringsheim's Theorem (see, e.g., [14, Theorem IV.6, p. 240]) is important in asymptotic enumeration where generating functions with non-negative coefficients are usually involved.

Lemma 2.1. Assume that $a_{m} \geq 0$ for all $m \geq 0$, and let the series expansion (2.1) have a finite radius of convergence $R$. Then the point $t=R$ is a singularity of the function $g(t)$.

The generating function $g_{\Theta}(t)$ in (1.4), constructed with the coefficients $\left(\theta_{m}\right)_{m \geq 1}$, plays a crucial role in this paper. Indeed, we will see that the asymptotic behaviour of the measure $\mathbb{P}_{\Theta}$ is determined by the analytic properties of the function $g_{\Theta}(z)$.

Recall that the cycle counts $C_{m}=C_{m}(\sigma)$ are defined as the number of cycles of length $m \in \mathbb{N}$ in the cycle decomposition of permutation $\sigma \in \mathfrak{S}_{n}$ (see the Introduction). The next well-known identity is a special case of the general Pólya's Enumeration Theorem [20, $\S 16$, p. 17] and the proof can be found for instance in [18, p. 5].

Lemma 2.2. Let $\left(a_{m}\right)_{m \in \mathbb{N}}$ be a sequence of (real or complex) numbers. Then one has the following (formal) power series expansion

$$
\exp \left(\sum_{m=1}^{\infty} \frac{a_{m} t^{m}}{m}\right)=\sum_{n=0}^{\infty} \frac{t^{n}}{n !} \sum_{\sigma \in \mathfrak{S}_{n}} \prod_{m=1}^{n} a_{m}^{C_{m}},
$$

where $C_{m}=C_{m}(\sigma)$ are the cycle counts. If either of the series in (2.2) is absolutely convergent then so is the other one.

We get immediately that

Corollary 2.3. Let $h_{n}$ be the normalisation constant in Definition 1.1 and $g_{\Theta}(t)$ be as in (1.4). We then have as formal power series in $t$

$$
\sum_{n=0}^{\infty} h_{n} t^{n}=\exp \left(g_{\Theta}(t)\right)
$$


2.2. Properties of $g_{\Theta}(t)$ in the case $\theta_{m}=\log ^{k}(m)$. In this section, we study the analytic properties of the function $g_{\Theta}(t)$ in the case $\theta_{m}=\log ^{k}(m)$. Inserting $\theta_{m}=\log ^{k}(m)$ into the definition of $g_{\Theta}(t)$ in (1.4), we obtain

$$
g_{\Theta}(t)=\sum_{m=1}^{\infty} \frac{\log ^{k} m}{m} t^{m} .
$$

This function has radius of convergence 1. A big part of our argumentation is based on the saddle-point method. For this we require the asymptotic behavior as $t \rightarrow 1$. Note that the function $g_{\Theta}(t)$ in (2.4) is a special case of the polylogarithm, see [14, §VI.8] and [13] as well as [10] for uses of the polylog in polynomial partitions. We thus summarize here only the properties we need and give only a sketch of the proofs. For a detailed proof, we refer to [14].

Lemma 2.4. Let $\theta_{m}$ be as in (1.3). Then $g_{\Theta}(t)$ is given by (2.4) and $g_{\Theta}(t)$ can be analytically continued to $\mathbb{C} \backslash[1, \infty]$. Further, there exists a polynomial $P$ with

$$
P(r)=\frac{r^{k+1}}{k+1}+\sum_{j=0}^{k} c_{j} r^{j}
$$

with $c_{j} \in \mathbb{R}$ for $0 \leq j \leq k$ such that

$$
g_{\Theta}\left(e^{-w}\right)=P(-\log (w))+O(w)
$$

for $w \rightarrow 0$ with $\arg (w) \leq \pi-\epsilon$ and $\epsilon>0$ arbitrary.

Equation (2.6) is related to 1.6 by inserting $w=-\log (z)$ and then expanding. Indeed, we have as $z \rightarrow 1$ with $|z|<1$ that

$$
-\log (-\log z)=-\log (-(z-1))+O(z-1) .
$$

Combining this with (2.6) gives

$$
\begin{aligned}
g_{\Theta}(z) & =P(-\log (-\log z))+O(z-1)=P(-\log (-(z-1))+O(z-1))+O(z-1) \\
& =P(-\log (1-z))+O\left((z-1)^{1-\delta}\right) \text { for } \delta>0 .
\end{aligned}
$$

Inserting this computation into the generating function of $h_{n}$ in (2.3), we indeed get (1.6). However, we will work with the expression $g_{\Theta}\left(e^{-w}\right)$ instead $g_{\Theta}(z)$ as this is more convenient in our computations.

Sketch of proof. The function $g_{\Theta}(t)$ has clearly radius of convergence 1 and is thus analytic for $|t|<1$. For the analytic continuation, one uses Lindelöfs integral representation of the polylogarithm, see for instance [14, IV.8, Page 237], namely

$$
g_{\Theta}(-t)=\frac{-1}{2 \pi i} \int_{1 / 2-i \infty}^{1 / 2+i \infty} \frac{\log ^{k}(s)}{s} \frac{t^{s} \pi}{\sin (\pi s)} d s .
$$

It is now easy to see that this integral is absolutely convergent for $t \in \mathbb{C} \backslash[0, \infty]$ and that it defines in $\mathbb{C} \backslash[0, \infty]$ an analytic function. Combining this with the fact that $g_{\Theta}(t)$ has radius of convergence 1 proves the first part of the lemma.

To compute the asymptotic behaviour of $g_{\Theta}\left(e^{-w}\right)$ as $w \rightarrow 0$, we use the Mellin transform, see for instance [14, §B.7]. Applying some elementary properties of the Mellin transform, we 
get immediately

$$
g_{\Theta}^{*}(s):=\int_{0}^{\infty} g_{\Theta}\left(e^{-w}\right) w^{s-1} d w=(-1)^{k} \zeta^{(k)}(s+1) \Gamma(s),
$$

where $\zeta^{(k)}(s)$ is the $k^{\prime}$ th derivative of the Riemann zeta function and $\Gamma$ is the Gamma function. Using the inverse Mellin transform, we obtain

$$
g_{\Theta}\left(e^{-w}\right)=\int_{1 / 2-i \infty}^{1 / 2+i \infty}(-1)^{k} \zeta^{(k)}(s+1) \Gamma(s) w^{-s} d s .
$$

We now shift the contour of integration to $\operatorname{Re}(s)=-3 / 2$. By doing this, we pick up poles at $s=0$ and at $s=-1$ so that

$$
\begin{aligned}
g_{\Theta}\left(e^{-w}\right)= & \int_{-3 / 2-i \infty}^{-3 / 2+i \infty}(-1)^{k} \zeta^{(k)}(s+1) \Gamma(s) w^{-s} d s \\
& +\operatorname{res}_{s=0}\left((-1)^{k} \zeta^{(k)}(s+1) \Gamma(s) w^{-s}\right)+\operatorname{res}_{s=-1}\left((-1)^{k} \zeta^{(k)}(s+1) \Gamma(s) w^{-s}\right) .
\end{aligned}
$$

We consider the Laurent expansion of $(-1)^{k} \zeta^{(k)}(s+1) \Gamma(s)$ around $s=0$ and get

$$
(-1)^{k} \zeta^{(k)}(s+1) \Gamma(s)=k ! s^{-2-k}+\sum_{j=0}^{k} d_{j} s^{-j-1}+O(1)
$$

for some $d_{j} \in \mathbb{R}, 0 \leq j \leq k$. Note that this Laurent expansion is independent of $w$. Using the Taylor expansion of $w^{-s}=e^{-s \log w}$ around $s=0$ then gives

$$
\begin{aligned}
\operatorname{res}_{s=0}\left((-1)^{k} \zeta^{(k)}(s+1) \Gamma(s) w^{-s}\right) & =(-1)^{k+1} \frac{\log ^{k+1}(w)}{k+1}+\sum_{j=0}^{k} d_{j}(-1)^{j} \frac{\log ^{j}(w)}{j !} \\
& =P(-\log (w))
\end{aligned}
$$

with $P(r)=\frac{1}{k+1} r^{k+1}+\sum_{j=0}^{k} d_{j} \frac{r^{j}}{j !}$. Thus the residue at $s=0$ has the form we are looking for. Since $\Gamma(s)$ has a simple pole with residue -1 at $s=-1$, we get that

$$
\operatorname{res}_{s=-1}\left((-1)^{k} \zeta^{(k)}(s+1) \Gamma(s) w^{-s}\right)=(-1)^{k+1} \zeta(0) w .
$$

The integral in (2.11) is well defined for all $w$ with $\arg (w) \leq \pi / 2-\epsilon$ since $|\Gamma(\sigma+i t)|=$ $O\left(t^{2} e^{-\frac{\pi}{2} t}\right)$ for $|t| \rightarrow \infty$ and $\sigma>-2$. A direct estimate then shows that this integral is of order $O\left(w^{3 / 2}\right)$. This shows that the above expansion in 2.6 is valid for $\arg (w) \leq \pi / 2-\epsilon$. To complete the proof, it remains to show that this expansion is also valid for $|\arg (w)| \leq \pi-\epsilon$. We omit this proof as it follows the same lines as in the proof of [13, Lemma 3].

Remark. One can easily relate the coefficients $c_{j}$ in Lemma 2.4 to the Laurent expansion of $\Gamma(s)$ around $s=0$. However, for our purpose it is enough to know that the $c_{j}$ are real numbers. Further, one can obtain with the above argumentation a complete asymptotic expansion of $g_{\Theta}\left(e^{-w}\right)$ and this asymptotic expansion is valid for $|\arg (w)| \leq \pi-\epsilon$. However, we do not need it here and thus will not prove it. Details can be found for instance in [14, §B.7] and [13].

2.3. Working assumptions on the cycle weights $\theta_{m}$. As mentioned in the Introduction, we are mainly interested in the cycle weights of the form $\theta_{m}=\log ^{k} m$. However, we require for our argumentation only some analytic properties of $g_{\Theta}(t)$. This allows us to work with more general cycle weights than $\theta_{m}=\log ^{k} m$. In view of Lemma 2.4 and the proof of Theorem 2.5 in Section 2.4, we will work with the following assumptions: 
- $\theta_{m} \geq 0$ for all $m \geq 1$,

- $g_{\Theta}(t)$ is holomorphic for $|t|<1$,

- $g_{\Theta}(t)$ is continuous in the punctured disc $\{|t| \leq 1, t \neq 1\}$ and

- we have as $w \rightarrow 0$ with $\operatorname{Re}(w) \geq 0$

$$
g_{\Theta}\left(e^{-w}\right)=P(-\log (w))+O(w),
$$

where $P(r)$ is a polynomial of degree $k+1$ with

$$
P(r)=\frac{r^{k+1}}{k+1}+\sum_{j=0}^{k} c_{j} r^{j} \text { with } c_{j} \in \mathbb{R} \text { for } 0 \leq j \leq k .
$$

A natural question at this point is: which cycle weights $\theta_{m}$ fulfill these assumptions? We know from Lemma 2.4 that this is the case for $\theta_{m}=\log ^{k} m$, but there are of course more. If a function $g_{\Theta}(t)$ fulfills the above assumptions then one can apply the method of singularity analysis to determine the asymptotic behaviour $\theta_{m}$, see [14][Page 387, (27)]. This then shows that the cycle weights $\theta_{m}$ must have the form

$$
\theta_{m}=\log ^{k}(m)+\sum_{j=0}^{k-1} a_{j} \log ^{j}(m)+o(1)
$$

for some $a_{j} \in \mathbb{R}$. We note that $(2.18)$ is not equivalent to the above assumptions on $g_{\Theta}(t)$. Indeed, we chose $\theta_{m}=\log ^{k}(m)+\log ^{-1}(m)$ then it is straight forward to see that (2.16) is not fulfilled. However, if the $o(1)$ in $(2.18)$ is replaced by $O\left(m^{-\epsilon}\right)$ for some $\epsilon>0$ then our assumptions on $g_{\Theta}(t)$ are fulfilled. Another natural question is if one can generalise the above assumptions. For instance if one can add a slowly varying function in the definition of the cycle weights as in [9]. However, this the topic of future research and will not be studied in this paper.

2.4. Asymptotic theorems. In this section, we develop complex-analytic tools for computing the asymptotics of the coefficient $h_{n}$ in the power series expansion of $\exp \left(g_{\Theta}(t)\right)$ (see (2.3) for the cycle weights $\theta_{m}$ fulfilling the assumptions in Section 2.3. More generally, it is useful to consider expansions of the function $\exp \left(v g_{\Theta}(t)\right)$, with some parameter $v>0$. We will see that the case $v=1$ is of primary importance, but we will need for instance in Theorem 3.2 also the behavior for $v \approx 1$ to deduce some limit theorems.

Theorem 2.5. Suppose $g_{\Theta}(t)$ fulfills the working assumptions in Section 2.3 Further, let $f(t)$ be a holomorphic function with radius of convergence strictly bigger than 1 and $f(1) \neq 0$. We then have for $v>0$

$$
\left[t^{n}\right]\left(f(t) \exp \left(v g_{\Theta}(t)\right)\right)=\frac{f(1) \exp \left(v P(r)+n e^{-r}\right)}{e^{r} \sqrt{2 \pi v P^{\prime \prime}(r)+2 \pi n e^{-r}}}\left(1+O\left(\log ^{-k / 2}(n)\right)\right),
$$

where $r$ is a solution of the equation

$$
v P^{\prime}(r)=n e^{-r}
$$

Furthermore, the error term in (2.19) is uniform in $v$ for $v \in\left[v_{1}, v_{2}\right]$, where $v_{1}, v_{2}$ are arbitrary, but fixed constants with $1 \leq v_{1}<v_{2}<\infty$.

We have $P^{\prime}(r) \sim r^{k}$ as $r \rightarrow \infty$ and thus 2.20) has a solution for $n$ large. Note that the solution $r$ is unique if $c_{j} \geq 0$ for all $j$. This does not have to be the case if some of the $c_{j}$ 
are negative. However, a straight forward computation shows that all solutions fulfill the same asymptotic expansion

$$
r=\log (n / v)-k \log \log (n / v)+O\left(\frac{\log \log (n)}{\log (n)}\right) \text { as } n \rightarrow \infty .
$$

From this, we immediately get

$$
\begin{aligned}
P(r) & =\frac{\log ^{k+1}(n)}{k+1}\left(1+O\left(\log ^{-1}(n)\right)\right), P^{\prime \prime}(r)=k \log ^{k-1}(n)\left(1+O\left(\log ^{-1}(n)\right)\right) \\
v P^{\prime}(r) & =n e^{-r}=v \log ^{k}(n)\left(1+O\left(\log ^{-1}(n)\right)\right) .
\end{aligned}
$$

For the proof of Theorem 2.5 we will use the saddle point method. Unfortunately, the function $g_{\Theta}(t)$ is in this situation not (log-)Hayman admissible (see [8] and [14, $\S$ VIII.5.]). We thus cannot use the standardized saddle point method, which is described for instance in [14, §VIII.5.]. We therefore use a slightly modified version. Also, we need an auxiliary result.

Lemma 2.6. Let $C>0$ be given. Let further $Q(x)=a_{d} x^{d}+\ldots+a_{0}$ be a real polynomial with $a_{d}>0$ and $d \geq 2$. We then have as $r \rightarrow \infty$

$$
\int_{C}^{r} \exp (Q(y)) d y=\frac{1}{Q^{\prime}(r)} \exp (Q(r))+O\left(\frac{1}{\left(Q^{\prime}(r)\right)^{2}} \exp (Q(r))\right) .
$$

Proof. We define $\delta:=\log \log (r)$. It is easy to see that we have for $r$ large enough

$$
\frac{1}{2^{d}} Q(r) \leq Q(r-\delta) \leq Q(r)-\frac{\delta}{2} Q^{\prime}(r) .
$$

We then split the integral in (2.24) into the integrals over $[C, r-\delta]$ and $[r-\delta, r]$. For $r$ large enough, $Q(y)$ attains its maximum in the interval $[C, r-\delta]$ at the point $r-\delta$. We thus use for the integral over $[C, r-\delta]$ the trivial estimate and get

$$
\left|\int_{C}^{r-\delta} \exp (Q(y)) d y\right| \leq r \exp (Q(r-\delta)) \leq \exp \left(Q(r)+\log (r)-\frac{\delta}{2} Q^{\prime}(r)\right) .
$$

By assumption, we have $\delta \cdot Q^{\prime}(r) / \log (r) \rightarrow \infty$ and thus $\log (r)-\frac{\delta}{2} Q^{\prime}(r) \leq-K \log (r)$ for $r$ large enough, where $K$ can be chosen arbitrary large. This implies that

$$
\int_{C}^{r-\delta} \exp (Q(y)) d y=O\left(\exp (Q(r)) r^{-K}\right) .
$$

For the integral over $[r-\delta, r]$, we use partial integration and a similar estimate as above to obtain

$$
\begin{aligned}
\int_{r-\delta}^{r} \exp (Q(y)) d y & =\frac{1}{Q^{\prime}(r)} e^{Q(y)}-\frac{1}{\left(Q^{\prime}(r)\right)^{2}} e^{Q(y)}+\int_{r-\delta}^{r} \frac{1}{\left(Q^{\prime}(y)\right)^{2}} e^{Q(y)} d y+O\left(e^{Q(r)} r^{-K}\right) \\
& =\frac{1}{Q^{\prime}(r)} e^{Q(y)}+O\left(\frac{1}{\left(Q^{\prime}(r)\right)^{2}} e^{Q(y)}\right) .
\end{aligned}
$$

This completes the proof.

Proof of Theorem 2.5. We begin with the case $f(t) \equiv 1$. Cauchy's integral formula gives

$$
I_{n}:=\left[t^{n}\right]\left(\exp \left(v g_{\Theta}(t)\right)\right)=\frac{1}{2 \pi i} \oint_{\gamma} \exp \left(v g_{\Theta}(t)\right) \frac{1}{t^{n+1}} d t
$$


where $\gamma$ is the circle $\gamma:=\left\{t=e^{-1 / 2} e^{i \varphi}, \varphi \in[-\pi, \pi]\right\}$. Applying the variable substitution $t=e^{-w}$, we get

$$
I_{n}=\frac{1}{2 \pi i} \int_{\gamma^{\prime}} \exp \left(v g_{\Theta}\left(e^{-w}\right)\right) e^{n w} d w
$$

with $\gamma^{\prime}:=\{t=1 / 2+i s, s \in[-\pi, \pi]\}$. Note that the integrand in (2.28) is $2 \pi i$ periodic. We thus can shift the contour $\gamma^{\prime}$ to the contour $\gamma^{\prime \prime}=\gamma_{1}^{\prime \prime} \cup \gamma_{2}^{\prime \prime} \cup \gamma_{3}^{\prime \prime}$ (see Figure 1) with

$$
\begin{aligned}
\gamma_{1}^{\prime \prime} & :=\left\{w=(-\pi+x) i, x \in\left[0, \pi-e^{-r}\right]\right\}, \\
\gamma_{2}^{\prime \prime} & :=\left\{w=e^{-r} e^{i \varphi}, \varphi \in[-\pi / 2, \pi / 2\},\right. \\
\gamma_{3}^{\prime \prime} & :=\left\{w=i x, x \in\left[e^{-r}, \pi\right]\right\},
\end{aligned}
$$

where $r$ is the solution of the equation 2.20 . We thus can write $I_{n}=I_{n, 1}+I_{n, 2}+I_{n, 3}$, where

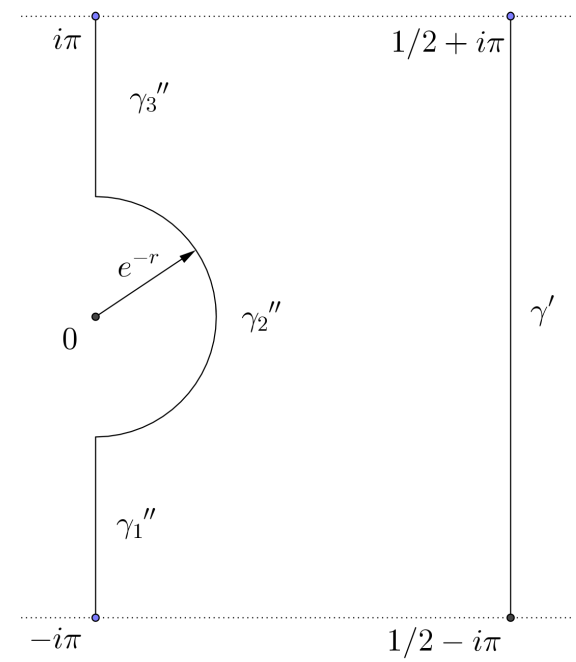

FIGURE 1. The contours $\gamma^{\prime}$ and $\gamma^{\prime \prime}$.

$I_{n, j}$ is the integral over $\gamma_{j}^{\prime \prime}$.

We begin by computing $I_{n, 2}$ with the saddle point method. We thus first take a look at the behaviour of the integrand in $I_{n, 2}$ for $\varphi$ around 0 . We use (2.16) and get

$$
g_{\Theta}\left(e^{-e^{-r} e^{i \varphi}}\right)=P(r-i \varphi)+O\left(e^{-r} e^{i \varphi}\right) .
$$

Expanding $P(r-i \varphi)$ around $\varphi=0$ gives

$$
P(r-i \varphi)=P(r)-i \varphi P^{\prime}(r)-\frac{1}{2} P^{\prime \prime}(r) \varphi^{2}+O\left(\varphi^{3} r^{k-2}\right) \text { as } \varphi \rightarrow 0 .
$$

We now split the integral $I_{n, 2}$ into the regions $[-\delta, \delta]$ and $[-\pi / 2, \pi / 2] \backslash[-\delta, \delta]$ for some $\delta>0$ small, which we determine below. We first take a look at the integral over $[-\delta, \delta]$. With 2.16 
we get

$$
\begin{aligned}
I_{n, 2, \delta}:= & \frac{e^{-r}}{2 \pi} \int_{-\delta}^{\delta} \exp \left(v g\left(e^{-e^{-r} e^{i \varphi}}\right)+n e^{-r} e^{i \varphi}+i \varphi\right) d \varphi \\
= & \frac{1}{2 \pi e^{r}} \int_{-\delta}^{\delta} \exp \left(v P(r-i \varphi)+n e^{-r} e^{i \varphi}+i \varphi+O\left(v e^{-r} e^{i \varphi}\right)\right) d \varphi \\
= & \frac{1}{2 \pi e^{r}} \int_{-\delta}^{\delta} \exp \left(v\left(P(r)-i \varphi P^{\prime}(r)-\frac{1}{2} P^{\prime \prime}(r) \varphi^{2}+O\left(\varphi^{3} r^{k-2}\right)\right)\right) \\
& \quad \times \exp \left(n e^{-r} e^{i \varphi}+i \varphi+O\left(e^{-r}\right)\right) d \varphi .
\end{aligned}
$$

Expanding $n e^{-r} e^{i \varphi}$ around $\varphi=0$ and using that we have $v P^{\prime}(r)=n e^{-r}$ by the definition of $r$ in 2.20), we obtain

$$
\begin{aligned}
I_{n, 2, \delta}= & \frac{\exp \left(v P(r)+n e^{-r}\right)}{2 \pi e^{r}} \\
& \times \int_{-\delta}^{\delta} \exp \left(-\frac{1}{2}\left(v P^{\prime \prime}(r)+n e^{-r}\right) \varphi^{2}\right) \exp \left(i \varphi+O\left(\varphi^{3}\left(r^{k-2}+n e^{-r}\right)\right)+O\left(e^{-r}\right)\right) d \varphi .
\end{aligned}
$$

We know from (2.21), 2.22) and (2.23) that

$$
r \sim \log (n), P^{\prime \prime}(r) \sim k \log ^{k-1}(n) \text { and } n e^{-r} \sim v \log ^{k}(n) \text { as } n \rightarrow \infty .
$$

Thus $n e^{-r}$ is dominating in the coefficients of $\varphi^{2}$ and $\varphi^{3}$ in the above expression for $I_{n, 2, \delta}$. We now define $\delta:=\delta(n, v)=\left(n e^{-r}\right)^{-5 / 12}$. Thus $\delta \rightarrow 0$ and

$$
\delta^{2}\left(v P^{\prime \prime}(r)+n e^{-r}\right) \rightarrow \infty \text { and } \delta^{3}\left(r^{k-2}+n e^{-r}\right) \rightarrow 0 .
$$

We therefore get

$$
I_{n, 2, \delta}=\frac{\exp \left(v P(r)+n e^{-r}\right)}{2 \pi e^{r}} \int_{-\delta}^{\delta} e^{-\frac{1}{2}\left(v P^{\prime \prime}(r)+n e^{-r}\right) \varphi^{2}}\left(1-i \varphi+O\left(\varphi^{2}+\varphi^{3} n e^{-r}+e^{-r}\right)\right) d \varphi .
$$

For notational convince, we write $b:=v P^{\prime \prime}(r)+n e^{-r}$. The function $\varphi e^{-\frac{b}{2} \varphi^{2}}$ is odd and thus we can remove the $i \varphi$ in the last equation. Using the variable substitution $x^{2}=b \varphi^{2}$, we get

$$
\begin{aligned}
& \int_{-\delta}^{\delta} e^{-\frac{b}{2} \varphi^{2}}\left(1+O\left(\varphi^{2}\right)+O\left(n e^{-r} \varphi^{3}\right)+O\left(e^{-r}\right)\right) d \varphi \\
= & \frac{1}{\sqrt{b}} \int_{-\delta \sqrt{b}}^{\delta \sqrt{b}} e^{-\frac{1}{2} x^{2}}\left(1+O\left(b^{-1} x^{2}\right)+O\left(n e^{-r} b^{-3 / 2} x^{3}\right)+O\left(e^{-r}\right)\right) d x \\
= & \frac{1}{\sqrt{b}}\left(\int_{-\infty}^{\infty} e^{-\frac{1}{2} x^{2}} d x+O\left(e^{-\delta \sqrt{b}}\right)\right)\left(1+O\left(b^{-1}\right)+O\left(n e^{-r} b^{-3 / 2}\right)+O\left(e^{-r}\right)\right) \\
= & \frac{\sqrt{2 \pi}}{\sqrt{b}}\left(1+O\left(\log ^{-k / 2}(n)\right)\right) .
\end{aligned}
$$

We thus obtain

$$
I_{n, 2, \delta}=\frac{\exp \left(v P(r)+n e^{-r}\right)}{e^{r} \sqrt{2 \pi\left(v P^{\prime \prime}(r)+n e^{-r}\right)}}\left(1+O\left(\log ^{-k / 2}(n)\right)\right)
$$


We now show that remaining parts of $I_{n, 2}$ and $I_{n, 1}, I_{n, 3}$ are all of lower order. We denote by $I_{n, 2}^{c}$ the remaining part of the $I_{n, 2}$, i.e. $I_{n, 2}^{c}=I_{n, 2}-I_{n, 2, \delta}$. For this, we use the inequalities

$$
\begin{aligned}
\cos (\varphi) & \leq 1-\varphi^{2} / 12 \text { for }|\varphi| \leq \pi \text { and } \\
\operatorname{Re}(P(r-i \varphi)) & \leq P(r)\left(1-\frac{k(\varphi / r)^{2}}{12}\right) \text { for } r \text { large and }|\varphi| \leq \pi .
\end{aligned}
$$

We thus get

$$
\begin{aligned}
\left|I_{n, 2}^{c}\right| & =2\left|\frac{1}{2 \pi e^{r}} \int_{\delta}^{\pi} \exp \left(v P(r-i \varphi)+n e^{-r} e^{i \varphi}+i \varphi+O\left(v e^{-r} e^{i \varphi}\right)\right) d \varphi\right| \\
& \ll e^{-r} \int_{\delta}^{\pi} \exp \left(v \operatorname{Re}(P(r-i \varphi))+n e^{-r} \cos (\varphi)\right) d \varphi \\
& \ll \exp \left(v P(r)+n e^{-r}\right) e^{-r} \int_{\delta}^{\pi} \exp \left(-\frac{k v P(r) r^{-2}+n e^{-r}}{12} \varphi^{2}\right) d \varphi .
\end{aligned}
$$

We now have $k v P(r) r^{-2}=O\left(\log ^{k-1} n\right)=o\left(n e^{-r}\right)$ and thus

$$
\begin{aligned}
\left|I_{n, 2}^{c}\right| & \ll \exp \left(v P(r)+n e^{-r}\right) e^{-r} \int_{\delta}^{\pi} \exp \left(-\frac{n e^{-r}}{24} \varphi^{2}\right) d \varphi \\
& \ll \frac{\exp \left(v P(r)+n e^{-r}\right)}{e^{r} \sqrt{n e^{-r}}} \int_{\delta \sqrt{n e^{-r}}}^{\infty} \exp \left(-\frac{x^{2}}{2}\right) d x \\
& \ll \frac{\exp \left(v P(r)+n e^{-r}\right)}{e^{r} \delta \sqrt{n e^{-r}}} e^{-\delta \sqrt{n e^{-r}}} .
\end{aligned}
$$

Inserting the definition of $\delta$ and the asymptotic behaviour of $n e^{-r}$ shows that $I_{n, 2}^{c}$ is of lower order. It remains to show that the integrals over $I_{n, 1}$ and $I_{n, 3}$ are also of lower order. The computations for both are almost the same and we thus only take a look at $I_{n, 3}$. We have

$$
\left|I_{n, 3}\right| \leq \frac{1}{2 \pi}\left|\int_{e^{-r}}^{\pi} \exp \left(v g_{\Theta}\left(e^{-i x}\right)+n i x\right) d x\right| \leq \frac{1}{2 \pi} \int_{e^{-r}}^{\pi} \exp \left(\operatorname{Re}\left(v g_{\Theta}\left(e^{-i x}\right)\right)\right) d x .
$$

We first consider the asymptotic behaviour of $g_{\Theta}\left(e^{-i x}\right)$ as $x \rightarrow 0$. Equation 2.16 gives

$$
g_{\Theta}\left(e^{-i x}\right)=P(-\log (x)-i \pi / 2)+O(x) .
$$

Using the Taylor expansion, we get for $x \rightarrow 0$

$$
\operatorname{Re}\left(g_{\Theta}\left(e^{-i x}\right)\right)=P(-\log (x))-P^{\prime \prime}(-\log (x)) \pi^{2} / 8+O\left(P^{(4)}(-\log (x))\right)+O(x) .
$$

Since $-\log (x) \geq 0$ for $x<1$, there exists a constant $0<c<1$ such that

$$
\left.\operatorname{Re}\left(g_{\Theta}\left(e^{-i x}\right) \leq P(-\log (x))-\frac{9}{8} P^{\prime \prime}(-\log (x)) \text { for all } x \in\right] 0, c\right] .
$$

We now split the integral into the integral over the regions $\left[e^{-r}, c\right]$ and $[c, \pi]$. By assumption, $g_{\Theta}(t)$ is continuous in the punctured disc $\{|t| \leq 1, t \neq 1\}$. We thus clearly have

$$
\frac{1}{2 \pi} \int_{c}^{\pi} \exp \left(\operatorname{Re}\left(v g_{\Theta}\left(e^{-i x}\right)\right)\right) d x=O(1) \text {. }
$$


Furthermore, we get with the above estimates and the variable substitution $y=-\log (x)$

$$
\begin{aligned}
\frac{1}{2 \pi} \int_{e^{-r}}^{c} \exp \left(\operatorname{Re}\left(v g_{\Theta}\left(e^{-i x}\right)\right)\right) d x & \leq \frac{1}{2 \pi} \int_{e^{-r}}^{c} \exp \left(v P(-\log (x))-\frac{9}{8} v P^{\prime \prime}(-\log (x))\right) d x \\
& =\frac{1}{2 \pi} \int_{-\log (c)}^{r} \exp \left(v P(y)-\frac{9}{8} v P^{\prime \prime}(y)\right) e^{-y} d y
\end{aligned}
$$

Thus we can apply Lemma 2.6 with $Q(y)=v P(y)-\frac{9}{8} v P^{\prime \prime}(y)-y$ and get

$$
\left|I_{n, 3}\right|=O\left(\frac{\exp \left(v P(r)-\frac{9}{8} v P^{\prime \prime}(r)-r\right)}{P^{\prime}(r)-\frac{9}{8} v P^{\prime \prime \prime}(r)-1}\right) \text {. }
$$

We now have to show that this is of lower order. Recall that the main term in the theorem is

$$
=\frac{\exp \left(v P(r)+n e^{-r}-r\right)}{\sqrt{2 \pi v P^{\prime \prime}(r)+2 \pi n e^{-r}}} \text {. }
$$

Using that

$$
r \sim \log (n / v), v P^{\prime}(r)=n e^{-r} \sim v \log ^{k}(n) \text { and } P^{\prime \prime}(r) \sim k \log ^{k-1}(n)
$$

immediately completes the proof for the case $f(t) \equiv 1$. The proof for $f(t)$ as in the theorem is almost the same as for $f(t) \equiv 1$. We thus describe only the necessary adjustments. In the integral $I_{n, 2, \delta}$, one has to use the Taylor expansion of $f(t)$ around one. It is straight forward to see that only the term $f(1)$ gives a relevant contribution. In the remaining integrals, we use the estimate $f(t)=O(1)$. This completes the proof.

We will see that we need in the Sections 3,4 and 5 also some slight generalizations of Theorem 2.5

Corollary 2.7. Let $g_{\Theta}(t)$ and $P(r)$ be as in Theorem 2.5. Let further $f(t)$ be a function such that such that

- $f(t)$ is holomorphic for $|t|<1$,

- $f(t)$ is continuous in the punctured disc $\{|t| \leq 1, t \neq 1\}$ and

- there is $a j \geq 0$ and a $c_{f} \in \mathbb{C}$ such that

$$
f\left(e^{-w}\right)=c_{f} \frac{(-\log (w))^{k}}{w^{j}}+O\left(\frac{(-\log (w))^{j-1}}{w^{j}}\right) \text { as } w \rightarrow 0, \operatorname{Re}(w) \geq 0 .
$$

We then have

$$
\left[t^{n}\right] f(t) \exp \left(g_{\Theta}(t)\right)=c_{f} r^{k} e^{j r} \frac{\exp \left(P(r)+n e^{-r}\right)}{\sqrt{2 \pi P^{\prime \prime}(r)+2 \pi n e^{-r}}}\left(1+O\left(\log ^{-1 / 2} n\right)\right),
$$

where $r$ is the solution of the equation

$$
P^{\prime}(r)=n e^{-r}
$$

Proof. We use the same notation as in the proof of Theorem 2.5 and describe only the necessary adjustments. We have

$$
I_{n}=\frac{1}{2 \pi i} \int_{\gamma} f(t) \exp \left(v g_{\Theta}(t)\right) \frac{1}{t^{n+1}} d t=\frac{1}{2 \pi i} \int_{\gamma^{\prime \prime}} f\left(e^{-w}\right) \exp \left(v g_{\Theta}\left(e^{-w}\right)\right) e^{n w} d w .
$$


We use that $\gamma_{2}^{\prime \prime}=\left\{w=e^{-r} e^{i \varphi}, \varphi \in[-\pi, \pi]\right\}$ and obtain

$$
I_{n, 2, \delta}=\frac{1}{2 \pi e^{r}} \int_{-\delta}^{\delta} f\left(e^{-e^{-r} e^{i \varphi}}\right) \exp \left(v P(r-i \varphi)+n e^{-r} e^{i \varphi}+i \varphi+O\left(v e^{-r} e^{i \varphi}\right)\right) d \varphi .
$$

As $f(t)$ has a singularity at $t=1$, one has to check if $f$ has a relevant influence to the saddle point equation. However, it is not difficult to see that we can use the same $r$ as in Theorem 2.5 Thus we immediately obtain that

$$
\begin{aligned}
I_{n, 2, \delta} & =f\left(e^{-e^{-r}}\right) \frac{\exp \left(P(r)+n e^{-r}\right)}{\sqrt{2 \pi P^{\prime \prime}(r)+2 \pi n e^{-r}}}\left(1+O\left(\log ^{-1 / 2} n\right)\right) \\
& =c_{f} r^{k} e^{j r} \frac{\exp \left(P(r)+n e^{-r}\right)}{\sqrt{2 \pi P^{\prime \prime}(r)+2 \pi n e^{-r}}}\left(1+O\left(\log ^{-1 / 2} n\right)\right) .
\end{aligned}
$$

The remaining parts of $I_{2}$ are of lower order. This completes the proof.

\section{ASYMPTOTIC STATISTICS OF CYCLES}

We apply in this section Theorem 2.5 to determine the asymptotic behaviour of various random variables on $\mathfrak{S}_{n}$.

3.1. Normalisation constant $h_{n}$. Recall that we have seen in Corollary 2.3 that

$$
\sum_{n=0}^{\infty} h_{n} t^{n}=\exp \left(g_{\Theta}(t)\right)
$$

where $h_{n}$ is the normalisation constant of the measure $\mathbb{P}_{\Theta}$ in Definition 1.1. We thus immediately get with Theorem 2.5 that

$$
h_{n}=\frac{\exp \left(P(r)+n e^{-r}\right)}{e^{r} \sqrt{2 \pi P^{\prime \prime}(r)+2 \pi n e^{-r}}}\left(1+O\left(\log ^{-k / 2}(n)\right)\right),
$$

where $P$ is as in Section 2.3 and $r$ is the solution of the equation $P^{\prime}(r)=n e^{-r}$.

3.2. Cycle counts. Our first result deals with the asymptotics of the cycle counts $C_{m}$ 's (i.e., the numbers of cycles of length $m \in \mathbb{N}$, respectively, in a random permutation $\sigma \in \mathfrak{S}_{n}$ ).

Theorem 3.1. Suppose that $\Theta=\left(\theta_{m}\right)_{m \in \mathbb{N}}$ fulfils the assumptions in Section 2.3 and that $\mathfrak{S}_{n}$ is endowed with $\mathbb{P}_{\Theta}$. We then have for each $b \in \mathbb{N}$ as $n \rightarrow \infty$

$$
\left(C_{1}, C_{2}, \cdots, C_{b}\right) \stackrel{d}{\rightarrow}\left(Y_{1}, \cdots, Y_{b}\right)
$$

with $Y_{1}, \cdots, Y_{b}$ independent Poisson distributed random variables with $\mathbb{E}\left[Y_{m}\right]=\frac{\theta_{m}}{m}$.

Theorem 3.1 shows that the asymptotic behaviour of cycles counts follows the typical pattern of random permutations with cycle weights. The more interesting question is of course the behaviour in the case when we replace the fixed $b$ in Theorem 3.1 by a $b=b(n)$ with $b(n) \rightarrow \infty$ as $n \rightarrow \infty$. We study this question in Section 5 .

Proof. Using Lemma 2.2 it is forthright to see that we have

$$
\sum_{n=0}^{\infty} h_{n} \mathbb{E}_{\Theta}\left[\exp \left(i \sum_{m=1}^{b} s_{m} C_{m}\right)\right] t^{n}=\exp \left(\sum_{m=1}^{b} \frac{\theta_{m}}{m}\left(e^{i s_{m}}-1\right) t^{m}\right) \exp \left(g_{\Theta}(t)\right)
$$


as formal power series in $t$. The details of this computation can be found for instance in [19. Theorem 3.1]. Theorem 2.5 with $v=1$ and Lévy's continuity theorem immediately complete the proof.

3.3. Total number of cycles. We denote by $K_{0 n}$ the total number of cycles in the cycle decomposition of $\sigma \in \mathfrak{S}_{n}$, i.e.

$$
K_{0 n}:=\sum_{m=1}^{n} C_{m}
$$

Theorem 3.2. Suppose that $\Theta=\left(\theta_{m}\right)_{m \in \mathbb{N}}$ fulfils the assumptions in Section 2.3 and that $\mathfrak{S}_{n}$ is endowed with $\mathbb{P}_{\Theta}$. We then have

$$
\frac{K_{0 n}-\mathbb{E}\left[K_{0 n}\right]}{\sqrt{\frac{\log ^{k+1}(n)}{k+1}}} \stackrel{d}{\rightarrow} \mathcal{N}(0,1)
$$

where $\mathcal{N}(0,1)$ is the standard normal distribution and $\mathbb{E}\left[K_{0 n}\right] \sim \frac{\log ^{k+1}(n)}{k+1}$.

Theorem 3.2 shows that the behavior of $K_{0 n}$ is very similar to the behaviour under uniform and Ewens measure. Indeed, if we insert (formally) $k=0$ into (3.6), we recover the behaviour of $K_{0 n}$ under the uniform measure. We have on the other hand in the case $\theta_{m}=m^{\gamma}$ that $\mathbb{E}\left[K_{0 n}\right] \sim \mathrm{C} n^{\frac{\gamma}{1+\gamma}}$ for some $C>0$, see [11]. We thus see that the studied model is (at least in many points) closer to the uniform and Ewens measure than the case $\theta_{m}=m^{\gamma}$.

Proof. We have for each $s \in \mathbb{C}$ as formal power series in $t$

$$
\mathbb{E}_{\Theta}\left[\exp \left(s K_{0 n}\right)\right]=\mathbb{E}_{\Theta}\left[\exp \left(s \sum_{m=1}^{n} C_{m}\right)\right]=\frac{1}{h_{n}}\left[t^{n}\right] \exp \left(e^{s} g_{\Theta}(t)\right) .
$$

This equation follows immediately from Lemma 2.2. The exact details of this computation can be found for instance in [19. Lemma 4.1]. Although the expressions in (3.7) holds for general $s \in \mathbb{C}$, we will calculate the asymptotic behaviour of the moment generating function of $K_{0 n}$ only on the positive half-line $s \geq 0$. Theorem 2.2 in [7] shows that this is enough to prove statement of the theorem. Let $r$ be the solution of $e^{s} P^{\prime}(r)=n e^{-r}$. Theorem 2.5 then gives

$$
\left[t^{n}\right] \exp \left(e^{s} g_{\Theta}(t)\right)=\frac{\exp \left(e^{s} P(r)+e^{s} P^{\prime}(r)\right)}{e^{r} \sqrt{2 \pi e^{s} P^{\prime \prime}(r)+2 \pi e^{s} P^{\prime}(r)}}\left(1+O\left(\log ^{-k / 2}(n)\right)\right) .
$$

We now define

$$
\widetilde{K_{0 n}}:=\frac{K_{0 n}-\mathbb{E}\left[K_{0 n}\right]}{\sqrt{\frac{\log ^{k+1}(n)}{k+1}}} .
$$

As 3.8 hold uniformly of $s$ bounded, we can replace $s$ by $\tilde{s}=\frac{s}{\sqrt{\frac{\log k+1(n)}{k+1}}}$. We thus get with a direct computation that

$$
\mathbb{E}\left[e^{s \widetilde{K_{0 n}}}\right]=\frac{1}{h_{n}}\left[t^{n}\right] \exp \left(e^{\tilde{s}} g_{\Theta}(t)-\tilde{s} \mathbb{E}\left[K_{0 n}\right]\right)=e^{s^{2} / 2}\left(1+O\left(\log ^{-k / 2}(n)\right)\right) .
$$

This completes the proof of the theorem. 
3.4. Lexicographic ordering of cycles. Often cycles in the cycle decomposition of a permutation are ordered by length. Another convenient way is to list the cycles (and their lengths) via the lexicographic ordering, that is, by tagging them with a suitable increasing subsequence of elements starting from 1.

Definition 3.3. For permutation $\sigma \in \mathfrak{S}_{n}$ decomposed as a product of cycles, let $L_{1}=L_{1}(\sigma)$ be the length of the cycle containing element $1, L_{2}=L_{2}(\sigma)$ the length of the cycle containing the smallest element not in the previous cycle, etc. The sequence $\left(L_{j}\right)$ is said to be lexicographically ordered.

Our next aim is to determine the asymptotic behaviour of the lexicographically ordered cycles lengths. For this we have to extend the assumptions in Section 2.3 a little bit. We assume in addition that we have for all $j \geq 1$

$$
g_{\Theta}^{(j)}\left(e^{-w}\right)=(j-1) !\left(\frac{e^{w}}{w}\right)^{j}(-\log (w))^{k}+O\left(\left(\frac{e^{w}}{w}\right)^{j}(-\log (w))^{k-1}\right),
$$

where $g_{\Theta}^{(j)}(t)$ is the $j$ 'th derivative of $g_{\Theta}$. If the function $g_{\Theta}(t)$ fulfills the assumptions in Section 2.3 and can be analytically extended beyond the punctured disc $\{|t| \leq 1, t \neq 1\}$ then the assumption (3.11) is automatically fulfilled. For concreteness, let us define the following region.

Definition 3.4. Let $1<R$ and $0<\phi<\frac{\pi}{2}$ be given. We then define the domain $\Delta_{0}$ as

$$
\Delta_{0}=\Delta_{0}(R, \phi)=\{t \in \mathbb{C} ;|t|<R, z \neq 1,|\arg (z-1)|>\phi\} .
$$

An illustration of $\Delta_{0}(R, \phi)$ can be found in Figure 2. We then have

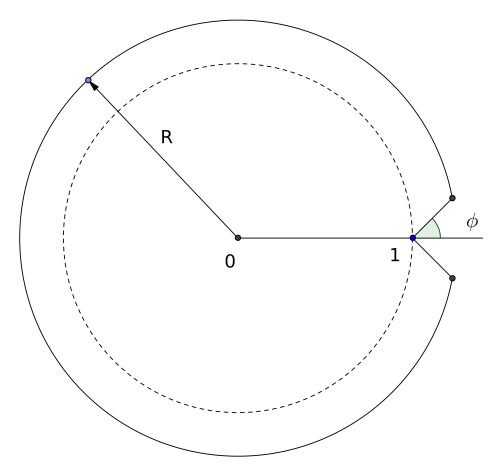

FIGURE 2. Illustration of $\Delta_{0}$

Lemma 3.5. Suppose that $\Theta=\left(\theta_{m}\right)_{m \in \mathbb{N}}$ fulfills the assumptions in Section 2.3 and that $g_{\Theta}(t)$ can be analytically extended to some domain $\Delta_{0}(R, \phi)$, with $\Delta_{0}(R, \phi)$ as in (3.12). Then the assumption (3.11) is fulfilled.

The lemma follows immediately with Cauchy's integral formula for higher order derivatives. Lemma 2.4 and 3.5 immediately imply that $g_{\Theta}(t)$ fulfils the assumption (3.11) if $\theta_{m}=\log ^{k}(m)$. We now can show 
Theorem 3.6. Suppose that $\Theta=\left(\theta_{m}\right)_{m \in \mathbb{N}}$ fulfils the assumptions in Section 2.3 and the assumption (3.11). If $\mathfrak{S}_{n}$ is endowed with $\mathbb{P}_{\Theta}$, we then have for each $b \in \mathbb{N}$

$$
\left(L_{1} \cdot \frac{\log ^{k}(n)}{n}, L_{2} \cdot \frac{\log ^{k}(n)}{n}, \ldots, L_{b} \cdot \frac{\log ^{k}(n)}{n}\right) \stackrel{d}{\longrightarrow}\left(E_{1}, E_{2}, \ldots, E_{b}\right),
$$

where $\left(E_{m}\right)_{m=1}^{b}$ are iid exponential distributed random variables with parameter 1.

Proof. We prove first the case $b=1$. We have

$$
\mathbb{P}\left[L_{1}=m\right]=\frac{\theta_{m}}{n} \frac{h_{n-m}}{h_{n}} .
$$

The proof of (3.14) can be found for instance in [11, Proposition 2.1]. We now claim that we have for each $j \in \mathbb{N}$

$$
\mathbb{E}_{\Theta}\left[\left(L_{1}-1\right)_{j}\right]=\frac{1}{n h_{n}}\left[t^{n}\right] t^{j} g_{\Theta}^{(j+1)}(t) \exp \left(g_{\Theta}(t)\right),
$$

where $(x)_{j}=x(x-1) \cdots(x-j+1)$ denotes the falling factorial. Indeed, using (3.14) gives

$$
\begin{aligned}
\mathbb{E}_{\Theta}\left[\left(L_{1}-1\right)_{j}\right] & =\frac{1}{h_{n}} \sum_{m=1}^{n}(m-1)_{j} \cdot \mathbb{P}\left[L_{1}=m\right]=\frac{1}{n h_{n}} \sum_{m=1}^{n}(m-1)_{j} \cdot \theta_{m} h_{n-m} \\
& =\frac{1}{n h_{n}}\left[t^{n}\right]\left(\sum_{m=1}^{n}(m-1)_{j} \cdot \theta_{m} t^{m}\right) \exp \left(g_{\Theta}(t)\right)=\frac{1}{n h_{n}}\left[t^{n}\right] t^{j} g_{\Theta}^{(j+1)}(t) \exp \left(g_{\Theta}(t)\right) .
\end{aligned}
$$

We now can use Corollary 2.7 together with assumption 3.11 to compute $\mathbb{E}_{\Theta}\left[\left(L_{1}-1\right)_{j}\right]$. We obtain

$$
\begin{aligned}
\mathbb{E}_{\Theta}\left[\left(L_{1}-1\right)_{j}\right] & =\frac{j !}{n} r^{k} e^{(j+1) r}(1+o(1))=j ! \frac{n^{j} r^{k}}{\left(n e^{-r}\right)^{j+1}}(1+o(1)) \\
& =j !\left(\frac{n}{r^{k}}\right)^{j}(1+o(1))=j !\left(\frac{n}{\log ^{k}(n)}\right)^{j}(1+o(1)),
\end{aligned}
$$

where we have used on the last line that $n e^{-r}=P^{\prime}(r) \sim r^{k}$ and $r \sim \log (n)$, see (2.21). This immediately implies with a simple induction that

$$
\mathbb{E}_{\Theta}\left[\left(\frac{L_{1} \cdot \log ^{k}(n)}{n}\right)^{j}\right]=j !\left(\frac{n}{\log ^{k}(n)}\right)^{j}(1+o(1)) .
$$

Since (3.16) holds for each $j \in \mathbb{N}$, we get that $L_{1} \cdot \frac{\log ^{k}(n)}{n}$ converges in distribution to exponential distributed random variables with parameter 1 . This completes the case $b=1$. The proof of the case $b>1$ is similarly and we thus omit it. However, the interested reader can find more details for instance in [6, Lemma 5.7] or in [22].

\section{LIMIT SHAPE}

We consider in this section the shape of Young diagrams associated to random permutations and study the typical behavior as $n \rightarrow \infty$ with respect to the measure $\mathbb{P}_{\Theta}$ under the assumptions in Section 2.3. We show that this shape converges to a limit shape and that fluctuations near a 
point of this limit shape behave like a normal random variable. In this section we shall mainly follow the techniques from [8]. We first define

$$
w_{n}(x)=\sum_{k \geq x} C_{k} .
$$

The function $w_{n}(x)=w_{n}(x, \sigma)$ is as a function in $x$ piecewise constant and right continuous. Further $w_{n}(x, \sigma)$ can be interpreted as the upper boundary of the Young diagram corresponding the cycle type of the permutation $\sigma$. A detailed illustration of this can be found in [8, Section 1].

The limit shape of the process $w_{n}(x)$ as $n \rightarrow \infty$ with of the respect to probability measures $\mathbb{P}_{\Theta}$ on $\mathfrak{S}_{n}$ (and sequences of positive real numbers $\bar{n}$ and $n^{*}$ with $\bar{n} \cdot n^{*}=n$ ) is understood as a function $w_{\infty}: \mathbb{R}^{+} \rightarrow \mathbb{R}^{+}$such that for each $\epsilon, \delta>0$

$$
\lim _{n \rightarrow+\infty} \mathbb{P}_{\Theta}\left[\left\{\sigma \in \mathfrak{S}_{n}: \sup _{x \geq \delta}\left|(\bar{n})^{-1} w_{n}\left(x n^{*}\right)-w_{\infty}(x)\right| \leq \epsilon\right\}\right]=1 .
$$

The assumption $\bar{n} \cdot n^{*}=n$ ensures that the area under the rescaled Young diagram is 1 . One of the most frequent choices is $\bar{n}=n^{*}=n^{1 / 2}$, however this is often not the optimal choice. The computations in Section 3 suggest that the length of a typical cycle has order of magnitude $n / r^{k}$. It is thus natural to choose

$$
n^{*}=\frac{n}{r^{k}} \text { and } \bar{n}=r^{k}
$$

with $r$ the solution (2.20). In order to avoid confusion, we write $r^{k}$ from now instead $\bar{n}$.

The next natural question is then whether fluctuations satisfy a central limit theorem, namely whether

$$
r^{-k} w_{n}\left(n^{*} x\right)-w_{\infty}(x)
$$

converges for a given $x$ (after centering and applying normalization) to a normal distribution. Also it is natural to ask if the process converges in distribution to a Gaussian process on the space of càdlàg functions. Of course the role of the probability measure on $\mathfrak{S}_{n}$ is important for that.

We first consider the behavior for a given $x>0$. We have

Theorem 4.1. Let $k \geq 3$ and suppose that $\mathbb{P}_{\Theta}$ fulfills the assumptions in Section 2.3. We then have the following results.

(1) The limit shape exists for the process $w_{n}(x)$ as $n \rightarrow \infty$ with the scaling $n^{*}$ as in 4.3 and $\bar{n}=r^{k}$. Further the limit shape is given by

$$
w_{\infty}(x)=\int_{x}^{\infty} u^{-1} e^{-u} d u .
$$

(2) The fluctuations at a point $x$ of the limit shape behave like

$$
\widetilde{w}_{n}(x):=\frac{w_{n}\left(x n^{*}\right)-r^{k}\left(w_{\infty}(x)+z_{n}(x)\right)}{r^{k / 2}} \stackrel{\mathcal{L}}{\longrightarrow} \mathcal{N}\left(0, \sigma_{\infty}^{2}(x)\right)
$$

with

$$
\text { and } z_{n}=O(1 / \log n) \text {. }
$$

$$
\sigma_{\infty}^{2}(x):=e^{-2 x}+w_{\infty}(x)
$$

Remark. The condition $k \geq 3$ is required in the estimates used for the error terms. However, we believe that this condition could be relaxed to $k \geq 1$ by a more detailed investigation of the corresponding error terms. 
We prove this theorem by computing the Laplace transform of $w_{n}(x)$. We have

Lemma 4.2. Let $k \geq 3$. We have for bounded $s \geq 0$ and with respect to $\mathbb{P}_{\Theta}$ as $n \rightarrow \infty$

$$
\mathbb{E}_{\Theta}\left[\exp \left(-s \widetilde{w}_{n}(x)\right)\right]=\sigma_{\infty}^{2}(x) \frac{s^{2}}{2}+O\left(r^{-k / 2} s^{3}\right)
$$

We will give the proof of Lemma 4.2 in Section 4.1. However, we show immediately that Lemma 4.2 implies Theorem 4.1. The structure of the proof is similar to the one appearing in [8], and we give the proof for the convenience of the reader.

Proof of Theorem 4.1] Theorem 2.2 in [7] shows that it is sufficient to compute the Laplace transform for $s \geq 0$ to establish the CLT. Therefore Lemma 4.2 immediately implies the second point of Theorem 4.1. Thus it remains to show that that $w_{\infty}(x)$ is the limit shape. Let $\epsilon>0$ be arbitrary and choose $0=x_{0}<x_{1}<\cdots<x_{\ell}$ such that

$$
w_{\infty}\left(x_{j+1}\right)-w_{\infty}\left(x_{j}\right)<\epsilon / 2 \text { for } 1 \leq j \leq \ell-1 \text { and } w_{\infty}\left(x_{\ell}\right)<\epsilon / 2 .
$$

We now claim that we have for each $x \in \mathbb{R}^{+}$

$$
\left|r^{-k} w_{n}\left(x n^{*}\right)-w_{\infty}(x)\right|>\epsilon \Longrightarrow \exists j \text { with }\left|r^{-k} w_{n}\left(x_{j} n^{*}\right)-w_{\infty}\left(x_{j}\right)\right|>\epsilon / 2 .
$$

Indeed, let us for consider first the case $r^{-k} w_{n}\left(x^{*}\right)-w_{\infty}(x)>\epsilon$. Clearly, there exists a $j$ such that $x_{j} \leq x \leq x_{j+1}$. Since $w_{n}(x)$ is a monotone decreasing function, we get immediately

$$
\begin{aligned}
r^{-k} w_{n}\left(x n^{*}\right)-w_{\infty}(x)>\epsilon & \Longrightarrow r^{-k} w_{n}\left(x_{j} n^{*}\right)-w_{\infty}(x)>\epsilon \\
& \Longrightarrow r^{-k} w_{n}\left(x_{j} n^{*}\right)-w_{\infty}\left(x_{j}\right)>\epsilon+w_{\infty}(x)-w_{\infty}\left(x_{j}\right) \\
& \Longrightarrow r^{-k} w_{n}\left(x_{j} n^{*}\right)-w_{\infty}\left(x_{j}\right)>\epsilon / 2 .
\end{aligned}
$$

The computation in the second case is similar. Using (4.6), we obtain

$$
\mathbb{P}_{\Theta}\left[\sup _{x \geq 0}\left|r^{k} w_{n}\left(x^{*}\right)-w_{\infty}(x)\right| \geq \epsilon\right] \leq \sum_{j=1}^{\ell} \mathbb{P}_{\Theta}\left[\left|r^{k} w_{n}\left(x_{j}^{*}\right)-w_{\infty}\left(x_{j}\right)\right| \geq \epsilon / 2\right]
$$

It now follows from (4.5) that each summand in 4.7 tends to 0 as $n \rightarrow \infty$. This completes the proof.

We are also interested in the joint behaviour at different points of the limit shape. For this, let $x_{\ell} \geq x_{\ell-1} \geq \cdots \geq x_{1} \geq 0$ be given. From computational point of view, it is easier to study the increments. We thus consider

$$
\mathbf{w}_{n}(\mathbf{x})=\left(w_{n}\left(x_{\ell}\right), w_{n}\left(x_{\ell-1}\right)-w_{n}\left(x_{\ell}\right), \ldots, w_{n}\left(x_{1}\right)-w_{n}\left(x_{2}\right)\right) .
$$

We now have

Theorem 4.3. For $\ell \geq 2$ and $x_{\ell} \geq x_{\ell-1} \geq \cdots \geq x_{1} \geq 0$, let

$$
\widetilde{\mathbf{w}}_{n}(\mathbf{x})=\left(\widetilde{w}_{n}\left(x_{\ell}\right), \widetilde{w}_{n}\left(x_{\ell-1}\right)-\widetilde{w}_{n}\left(x_{\ell}\right), \ldots, \widetilde{w}_{n}\left(x_{1}\right)-\widetilde{w}_{n}\left(x_{2}\right)\right)
$$

with $\widetilde{w}_{n}$ as in Theorem 4.1. Set $x_{\ell+1}=+\infty$. We then have for $1 \leq j<i<\ell$

$$
\begin{aligned}
\widetilde{w}_{\infty}\left(x_{i}, x_{j}\right) & :=\lim _{n \rightarrow+\infty} \operatorname{Cov}\left(\widetilde{w}_{n}\left(x_{j}\right)-\widetilde{w}_{n}\left(x_{j+1}\right), \widetilde{w}_{n}\left(x_{i}\right)-\widetilde{w}_{n}\left(x_{i+1}\right)\right) \\
& =\left(e^{-x_{j}}-e^{-x_{j+1}}\right)\left(e^{-x_{i}}-e^{-x_{i+1}}\right) .
\end{aligned}
$$


The proof of this theorem is given in Section 4.2

We have seen in Theorem 3.1 that the cycle counts $C_{1}, \ldots, C_{b}$ converge to independent Poisson random variables $Y_{1}, \ldots, Y_{b}$. For the uniform and Ewens measure, this remains true as long as $b=o(n)$, see [2]. However, Theorem 4.3 now shows that the increments in (4.9) are not independent in the limit. Thus a similar result can hold in the considered model at most for $b=o\left(n / \log ^{k}(n)\right)$. This will be the topic of Section 5 .

Theorem 4.3 can be extended to a functional CLT.

Theorem 4.4. The process $\widetilde{w}_{n}: \mathbb{R}^{+} \rightarrow \mathbb{R}$ (see Theorem 4.1) converges weakly with respect to $\mathbb{P}_{\Theta}$ as $n \rightarrow \infty$ to a continuous Gaussian process $\widetilde{w}_{\infty}: \mathbb{R}^{+} \rightarrow \mathbb{R}$. Explicitly, we have $\widetilde{w}_{\infty}(x) \sim \mathcal{N}\left(0,\left(\sigma_{\infty}(x)\right)^{2}\right)$ and covariance structure is given in Theorem 4.3 In particular, the increments are not independent.

The proof of this theorem is given in Section 4.3 .

4.1. Proof of Lemma 4.2, We begin with some preparations. We have

Lemma 4.5 ([8, Lemma 4.1]). We have for $x \geq 0$ and $s \in \mathbb{C}$

$$
\mathbb{E}_{\Theta}\left[\exp \left(-s w_{n}(x)\right)\right]=\frac{1}{h_{n}}\left[t^{n}\right]\left[\exp \left(g_{\Theta}(t)+\left(e^{-s}-1\right) \sum_{m=\lfloor x\rfloor}^{n} \frac{\theta_{m}}{m} t^{m}\right)\right] .
$$

Furthermore, we need

Lemma 4.6. Let $r$ be as in 2.20), $n^{*}$ be as in (4.3), $v=O\left(r^{-k / 2}\right), q>0, j \in \mathbb{Z}$ and $x>0$. We then define $r^{\prime}:=r+v$ and get

$$
\sum_{m \geq x n^{*}} \theta_{m} m^{j} \exp \left(-m q e^{-r^{\prime}}\right)=r^{k}\left(e^{r}\right)^{j+1} \int_{x}^{\infty} u^{j} \exp (-q u) d u+O\left(r^{k-1 / 2}\left(e^{r}\right)^{j+1}\right) .
$$

Proof. We know from (2.18) that $\theta_{m}$ has the form

$$
\theta_{m}=\log ^{k}(m)+\sum_{j=0}^{k-1} a_{j} \log ^{j}(m)+o(1) \text { for some } a_{j} \in \mathbb{R} .
$$

Thus is is sufficient to study the case $\theta_{m}=\log ^{k}(m)$ and to show that

$$
\sum_{m \geq x n^{*}} \log ^{k}(m) m^{j} \exp \left(-m q e^{-r^{\prime}}\right)=r^{k}\left(e^{r}\right)^{j+1} \int_{x}^{\infty} u^{j} e^{-q u} d u+O\left(r^{k-1 / 2}\left(e^{r}\right)^{j+1}\right) .
$$

We apply Euler's summation formula to the sum on the LHS in 4.12) with $\theta_{m}=\log ^{k}(m)$ and $f(y)=\log ^{k}(y) y^{j} \exp \left(-y q e^{-r^{\prime}}\right)$. This gives

$$
\begin{aligned}
\sum_{m \geq x n^{*}} \log ^{k}(m) m^{j} \exp \left(-m q e^{-r^{\prime}}\right)= & \int_{x n^{*}}^{\infty} \log ^{k}(y) y^{j} \exp \left(-y q e^{-r^{\prime}}\right) d y \\
& +\int_{x n^{*}}^{\infty}(y-\lfloor y\rfloor) f^{\prime}(y) d y-f\left(x n^{*}\right)\left(x n^{*}-\left[x n^{*}\right]\right)
\end{aligned}
$$


with $\lfloor y\rfloor=\max \{m \in \mathbb{N} ; m \leq y\}$. We first look at the integral

$$
\int_{x n^{*}}^{\infty} \log ^{k}(y) y^{j} \exp \left(-y q e^{-r^{\prime}}\right) d y
$$

We now use the variable substitution $u=y e^{-r^{\prime}}$ and get

$$
\begin{aligned}
\int_{x n^{*}}^{\infty} \log ^{k}(y) y^{j} \exp \left(-y q e^{-r^{\prime}}\right) d y & =\left(e^{r^{\prime}}\right)^{j+1} \int_{e^{-r^{\prime}} x n^{*}} \log ^{k}\left(u e^{r^{\prime}}\right) u^{j} \exp (-q u) d u \\
& =\left(e^{r^{\prime}}\right)^{j+1} \int_{e^{-r^{\prime}} x n^{*}}\left(\log (u)+r^{\prime}\right)^{k} u^{j} \exp (-q u) d u .
\end{aligned}
$$

Using that $n^{*}=n / r^{k}$ and that $P^{\prime}(r)=n e^{-r}$, we immediately obtain that

$$
\int_{x n^{*}}^{\infty} \log ^{k}(y) y^{j} \exp \left(-y e^{-r}\right) d y=r^{k}\left(e^{r}\right)^{j+1} \int_{x} u^{j} \exp (-q u) d u+O\left(r^{k-1 / 2}\left(e^{r}\right)^{j+1}\right) .
$$

This gives the desired asymptotic behaviour. We thus have to show that the remaining terms in (4.15) are of lower order. We have

$$
f^{\prime}(y)=\left(1+j \log (y)-y q e^{-r^{\prime}} \log (y)\right) \log ^{k-1}(y) y^{j-1} \exp \left(-y q e^{-r^{\prime}}\right) .
$$

Thus we can use the same computation as for the main term for the integral over $f^{\prime}(y)$ in (4.15) and immediately get that it is of lower order. Further, inserting the definition of $n^{*}$ into $f\left(x n^{*}\right)\left(x n^{*}-\left[x n^{*}\right]\right)$ also shows that it is of lower order.

We are now able to prove Lemma 4.2

Proof of Lemma 4.2. We use the definition of $\widetilde{w}_{n}(x)$ in (4.5) and obtain

$$
\mathbb{E}_{\Theta}\left[\exp \left(-s \widetilde{w}_{n}(x)\right)\right]=\exp \left(s r^{k / 2}\left(w_{\infty}(x)+z_{n}(x)\right)\right) \mathbb{E}_{\Theta}\left[\exp \left(-s^{*} w_{n}\left(x n^{*}\right)\right)\right]
$$

with $w_{n}(x)$ as in (4.1), $n^{*}$ as in (4.3) and $s^{*}:=s r^{-k / 2}$. Thus it is enough to compute the asymptotic behaviour of $\mathbb{E}_{\Theta}\left[\exp \left(-s^{*} w_{n}\left(x n^{*}\right)\right)\right]$. We now use Lemma 4.5 and replace $x$ by $x n^{*}$ and $s$ by $s^{*}$ in (4.11). This then gives

$$
h_{n} \mathbb{E}_{\Theta}\left[\exp \left(-s^{*} w_{n}\left(x n^{*}\right)\right)\right]=\left[t^{n}\right]\left[\exp \left(g_{\Theta}(t)+\left(e^{-s^{*}}-1\right) \sum_{m=\left\lfloor x n^{*}\right\rfloor}^{n} \frac{\theta_{m}}{m} t^{m}\right)\right] .
$$

The natural approach would be now to use Theorem 2.5 to compute the asymptotic behaviour of the last expression. Unfortunately, the additional term

$$
M_{n}(t):=\left(e^{-s^{*}}-1\right) \sum_{m=\left\lfloor x n^{*}\right\rfloor}^{n} \frac{\theta_{m}}{m} t^{m}
$$

in (4.18) has a relevant influence to the saddle point. We thus cannot directly use Theorem 2.5 but we can adjust the proof of Theorem 2.5 . We will focus here only on the necessary adjustments.

As in the proof of Theorem 2.5, we can rewrite $(4.20)$ as

$$
I_{n}:=\frac{1}{2 \pi i} \int_{\gamma^{\prime \prime}} \exp \left(g_{\Theta}\left(e^{-w}\right)+M_{n}\left(e^{-w}\right)\right) e^{n w} d w
$$

where $\gamma^{\prime \prime}$ is the same contour as in Figure 1, but we replace in $\gamma_{2}^{\prime \prime}$ the $r$ with a $r^{\prime}$, see below. 
We now use the same splitting of $I_{n}$ as in the proof of Theorem 2.5 and write

$$
I_{n}=I_{n, 2, \delta}+I_{n, 2}^{c}+I_{n, 1}+I_{n, 3} .
$$

The remaining proof is now structured as follows: We first determine $r^{\prime}$ so that we can apply the saddle point method, then we evaluate $I_{n, 2, \delta}$ and finally we show that the remaining parts are of lower order.

We now insert the definition of $\gamma_{2}^{\prime \prime}$ into of $I_{n, 2, \delta}$, see Figure 8 , and use that $g_{\Theta}\left(e^{-w}\right)=$ $P(-\log (w))+O(w)$ to obtain

$$
\begin{aligned}
I_{n, 2, \delta} & =\frac{1}{e^{r^{\prime}} 2 \pi} \int_{-\delta}^{\delta} \exp \left(f(\varphi)+i \varphi+O\left(e^{-r^{\prime}} e^{i \varphi}\right)\right) d \varphi \\
\text { with } \quad f(\varphi) & :=P\left(r^{\prime}-i \varphi\right)+n e^{-r^{\prime}} e^{i \varphi}+M_{n}\left(e^{-e^{-r^{\prime}} e^{i \varphi}}\right) .
\end{aligned}
$$

We now write $f(\varphi)=f(0)+i a\left(r^{\prime}\right) \varphi-b\left(r^{\prime}\right) \frac{\varphi^{2}}{2}+R_{n}\left(\varphi, r^{\prime}\right)$. In order to be able to apply the saddle point method, we have to find $r^{\prime}=r^{\prime}(n, x)$ and $\delta=\delta(n, x)$ with

$$
b\left(r^{\prime}\right) \delta^{2} \rightarrow \infty, \delta \rightarrow 0, a\left(r^{\prime}\right)=o\left(\sqrt{b\left(r^{\prime}\right)}\right) \text { and } R_{n}\left(\varphi, r^{\prime}\right)=o\left(\varphi^{3} \delta^{-3}\right) .
$$

We now claim that we can choose

$$
r^{\prime}=r+v \text { with } v:=\frac{\left(e^{-s^{*}}-1\right) r^{k} e^{-x}}{n e^{-r}} \text { and } \delta=\left(n e^{-r}\right)^{-5 / 12},
$$

where $r$ is the solution of the equation $P^{\prime}(r)=n e^{-r}$. We now have

$$
a\left(r^{\prime}\right)=-P^{\prime}\left(r^{\prime}\right)+n e^{-r^{\prime}}-e^{-r^{\prime}} e^{-e^{-r^{\prime}}} M_{n}^{\prime}\left(e^{-e^{-r^{\prime}}}\right)
$$

Since $s^{*}=s r^{-k / 2}$ and $r \sim \log (n)$, see (2.21), we obtain $v \sim e^{-x} s r^{-k / 2}=O\left(r^{-k / 2}\right)$. By assumption, $P(r)$ has degree $k+1$ and $P^{\prime}(r)=n e^{-r}$. We thus have

$$
\begin{aligned}
-P^{\prime}\left(r^{\prime}\right)+n e^{-r^{\prime}} & =-P^{\prime}(r+v)+n e^{-r-v}=-P^{\prime}(r)+n e^{-r}+v n e^{-r}+O\left(r^{k / 2-1}\right) \\
& =v n e^{-r}+O\left(r^{k / 2-1}\right) .
\end{aligned}
$$

On the other hand, we get with Lemma 4.6 that

$$
\begin{aligned}
e^{-r^{\prime}} e^{-e^{-r^{\prime}}} M_{n}^{\prime}\left(e^{-e^{-r^{\prime}}}\right) & =\left(e^{-s^{*}}-1\right) e^{-r^{\prime}} \sum_{m=\left\lfloor x n^{*}\right\rfloor}^{n} \theta_{m} e^{-m e^{-r^{\prime}}} \\
& =\left(e^{-s^{*}}-1\right) r^{k} \int_{x}^{\infty} e^{-u} d u+O\left(r^{k / 2-1}\right)=\left(e^{-s^{*}}-1\right) e^{-x} r^{k}+O\left(r^{k / 2-1}\right) .
\end{aligned}
$$

Combining these two equations with the definition of $v$, we get that $a\left(r^{\prime}\right)=O\left(r^{k / 2-1}\right)$. In a similar way, we get $b\left(r^{\prime}\right)=P^{\prime \prime}(r)+n e^{-r}+O\left(r^{k / 2}\right) \sim r^{k}$ and that $R_{n}\left(\varphi, r^{\prime}\right)=O\left(r^{k} \varphi^{3}\right)$. This implies $b\left(r^{\prime}\right) \sim r^{k}, a\left(r^{\prime}\right)=o\left(\sqrt{b\left(r^{\prime}\right)}\right), b\left(r^{\prime}\right) \delta^{2} \rightarrow \infty$ and $R_{n}\left(\varphi, r^{\prime}\right)=o\left(\delta^{-3} \varphi^{3}\right)$. We thus can apply the saddle point method with $r^{\prime}$ and use the same computation as in 2.33). We obtain with $f$ as in (4.21) that

$$
I_{n, 2, \delta}=\frac{\exp (f(0))}{e^{r^{\prime}} \sqrt{2 \pi b\left(r^{\prime}\right)}}\left(1+O\left(\frac{a\left(r^{\prime}\right)}{\sqrt{b\left(r^{\prime}\right)}}\right)\right)=\frac{\exp (-v+f(0))}{e^{r} \sqrt{2 \pi\left(P^{\prime \prime}(r)+n e^{-r}\right)}}\left(1+O\left(r^{-1}\right)\right) .
$$

We now have to determine $f(0)$. 
We first look at $v$. We use $n e^{-r}=P^{\prime}(r)$ and $s^{*}=s r^{-k / 2}$ and get

$$
v=\frac{\left(e^{-s^{*}}-1\right) r^{k} e^{-x}}{n e^{-r}}=e^{-x}\left(-\frac{s}{r^{k / 2}}+\frac{s^{2}}{2 r^{k}}+O\left(s^{3} r^{-3 k / 2}\right)\right)(1+O(1 / r)) .
$$

Inserting this into $P\left(r^{\prime}\right)$ and using $r^{\prime}=r+v$ gives

$$
\begin{aligned}
P\left(r^{\prime}\right) & =P(r+v)=P(r)+P^{\prime}(r) v+\frac{1}{2} P^{\prime \prime}(r) v^{2}+O\left(v^{3} \log ^{k-2} n\right) \\
& =P(r)-e^{-x}(1+O(1 / r)) r^{-k / 2} s+e^{-x}(1+O(1 / r)) \frac{s^{2}}{2}+O\left(s^{3} r^{-k / 2}\right) .
\end{aligned}
$$

Furthermore, we have

$$
\begin{aligned}
n e^{-r^{\prime}} & =n e^{-r} e^{-v}=n e^{-r}\left(1-v+v^{2} / 2+O\left(v^{3}\right)\right) \\
& =n e^{-r}+e^{-x}(1+O(1 / r)) r^{k / 2} s+\left(e^{-2 x}-e^{-x}\right)(1+O(1 / r)) \frac{s^{2}}{2}+O\left(s^{3} r^{-k / 2}\right)
\end{aligned}
$$

and get with 4.19) and Lemma 4.6

$$
\begin{aligned}
M_{n}\left(e^{-e^{-r^{\prime}}}\right) & =\left(e^{-s^{*}}-1\right)\left(r^{k} \int_{x}^{\infty} u^{-1} e^{-u} d u+O\left(r^{k-1}\right)\right)(1+O(1 / r)) \\
& =w_{\infty}(x)\left(-r^{k / 2} s+s^{2} / 2+O\left(s^{3} r^{-k / 2}\right)\right)(1+O(1 / r))
\end{aligned}
$$

with $w_{\infty}(x)$ as in (4.4). Combining (4.25), (4.26) and (4.27), we obtain

$$
\begin{aligned}
I_{2, \delta}= & \frac{\exp \left(P(r)+n e^{-r}\right)}{e^{r} \sqrt{2 \pi\left(P^{\prime \prime}(r)+n e^{-r}\right)}}\left(1+O\left(r^{-1}\right)\right) \\
& \times \exp \left(-\left(w_{\infty}(x)+z_{n}(x)\right) r^{k / 2} s+\left(e^{-2 x}+w_{\infty}(x)\right)\left(1+O(1 / r) \frac{s^{2}}{2}+O\left(s^{3} r^{-k / 2}\right)\right)\right.
\end{aligned}
$$

with $z_{n}(x)=O(1 / r)=O(1 / \log n)$. Using Theorem 2.5, we immediately get that

$$
\frac{I_{2, \delta}}{h_{n}} \cdot \exp \left(w_{\infty}(x)(1+O(1 / r)) r^{k / 2} s\right) \longrightarrow \exp \left(\left(e^{-2 x}+w_{\infty}(x)\right) \frac{s^{2}}{2}\right) .
$$

Comparing this with 4.17, we immediately see that $\frac{I_{2, \delta}}{h_{n}}$ has the predicted behaviour of $\mathbb{E}_{\Theta}\left[\exp \left(-s \widetilde{w}_{n}(x)\right)\right]$. Thus the proof is complete if we can show that the remaining integrals are of lower order.

We consider next the integral $I_{n, 2}^{c}$. We split this integral into the integrals over the intervals $\left[\delta, \log ^{-2} r\right]$ and $\left[\log ^{-2} r, \pi / 2\right]$. We begin with the first interval. We get with Lemma 4.6 that

$$
\operatorname{Re}\left(\sum_{m=\left\lfloor x n^{*}\right\rfloor}^{n} \frac{\theta_{m}}{m} e^{-m e^{-r^{\prime}} e^{i \varphi}}\right)=\sum_{m=\left\lfloor x n^{*}\right\rfloor}^{n} \frac{\theta_{m}}{m} e^{-m e^{-r^{\prime}} \cos (\varphi)}=r^{k} \int_{x}^{\infty} \frac{e^{-\cos (\varphi) u}}{u} d u+O\left(r^{k-1 / 2}\right) .
$$

Splitting the last integral into two integrals over $[x, 2 k \log r]$ and $[2 k \log r, \infty]$, it is straight forward to see that

$$
\int_{x}^{\infty} \frac{e^{-\cos (\varphi) u}}{u} d u=\int_{x}^{\infty} \frac{e^{-u}}{u} d u+O\left(\varphi^{2} \int_{x}^{\infty} \frac{e^{-u}}{u}\right)+O\left(r^{-k}\right) .
$$

Using the definition of $M_{n}(t)$ in (4.19) and that $s^{*}=s r^{-k / 2}$, we have for $|\varphi| \leq \log ^{-2} r$

$$
\operatorname{Re}\left(M_{n}\left(e^{-e^{-r^{\prime}} e^{i \varphi}}\right)\right)=-s r^{k / 2}\left(w_{\infty}(x)+z_{n}(x)\right)+O\left(r^{k / 2} \varphi^{2}\right)+O(1) .
$$


Using the definition of $I_{n, 2}^{c}$ and $f$ in (4.21) and the estimates in 2.36, we obtain as in 2.37) and (2.38) that

$$
\begin{aligned}
& \quad\left|\frac{1}{2 \pi e^{r}} \int_{\delta}^{\log ^{-2} r} \exp (f(\varphi)) d \varphi\right| \ll e^{-r} \int_{\delta}^{\log ^{-2} r} \exp (\operatorname{Re}(f(\varphi))) d \varphi \\
& \ll \exp \left(P(r)+n e^{-r}-s r^{k / 2}\left(w_{\infty}(x)+z_{n}(x)\right)\right) e^{-r} \int_{\delta}^{\log ^{-2} r} \exp \left(-\frac{k P(r) r^{-2}+n e^{-r}}{24} \varphi^{2}\right) d \varphi \\
& \ll \frac{\exp \left(P(r)+n e^{-r}-s r^{k / 2}\left(w_{\infty}(x)+z_{n}(x)\right)\right)}{e^{r} \delta \sqrt{n e^{-r}}} e^{-\delta \sqrt{n e^{-r}}} .
\end{aligned}
$$

Thus this part of $I_{n, 2}^{c}$ is indeed of lower order. For the interval $\left[\log ^{-2} r, \pi / 2\right]$, we use that

$$
\operatorname{Re}\left(\left(e^{-s^{*}}-1\right) \sum_{m=\left\lfloor x n^{*}\right\rfloor}^{n} \frac{\theta_{m}}{m} e^{-m e^{-r^{\prime}} e^{i \varphi}}\right)=O\left(r^{k / 2+1}\right) .
$$

Using again the same argument as in 2.37) and 2.38, we obtain

$$
\begin{aligned}
& \left|\frac{1}{2 \pi e^{r}} \int_{\log ^{-2} r}^{\pi} \exp (f(\varphi)) d \varphi\right| \ll e^{-r} \int_{\log ^{-2} r}^{\pi} \exp (\operatorname{Re}(f(\varphi))) d \varphi \\
& \ll \exp \left(P(r)+n e^{-r}+O\left(r^{k / 2+1}\right)\right) e^{-r} \int_{\log ^{-2} r}^{\pi} \exp \left(-\frac{k P(r) r^{-2}+n e^{-r}}{24} \varphi^{2}\right) d \varphi \\
& \ll \frac{\exp \left(P(r)+n e^{-r}+O\left(r^{k / 2+1}\right)\right)}{e^{r} \sqrt{n e^{-r}}} \int_{\sqrt{n e^{-r}} \log ^{-2} r}^{\infty} \exp \left(-\frac{x^{2}}{2}\right) d x \\
& \ll \frac{\exp \left(P(r)+n e^{-r}+O\left(r^{k / 2+1}\right)\right)}{e^{r} \delta \sqrt{n e^{-r}}} e^{-n e^{-r} \log ^{-4} r} .
\end{aligned}
$$

We now have $n e^{-r} \log ^{-4} r \sim r^{k} \log ^{-4} r>r^{k / 2+1}$ since $k \geq 3$. This implies that this part of $I_{2, \delta}^{c}$ is also of lower order. Note that this inequality is the origin of the assumption $k \geq 3$ in this section. It remains to consider the integral $I_{3}$. Here we use also the bound (4.29) and the fact that $k \geq 3$. The computations closely parallel those of the proof of Theorem 2.5 and we may thus safely omit them. This completes the proof.

4.2. Proof of Theorem 4.3. The proof of Theorem 4.3 has the same ingredients as the proof of Theorem 4.1. We thus give only a sketch of the proof and highlight the necessary adjustments.

As for Theorem 4.1, we compute the Laplace transform of $\mathbf{w}_{n}(\mathbf{x})$. We begin with the generating function. We have

Lemma 4.7 ([8, Lemma 4.2]). We have for $\boldsymbol{x}=\left(x_{1}, \ldots, x_{\ell}\right) \in \mathbb{R}^{\ell}$ with $x_{\ell} \geq x_{\ell-1} \geq \cdots \geq$ $x_{1} \geq 0$ and $\boldsymbol{s}=\left(s_{1}, \ldots, s_{\ell}\right) \in \mathbb{C}^{\ell}$

$$
\mathbb{E}_{\Theta}\left[\exp \left(-\left\langle\boldsymbol{s}, \boldsymbol{w}_{n}(\boldsymbol{x})\right\rangle\right)\right]=\frac{1}{h_{n}}\left[t^{n}\right]\left[\exp \left(g_{\Theta}(t)+\sum_{j=1}^{\ell}\left(e^{-s_{j}}-1\right) \sum_{k=\left\lfloor x_{j}\right\rfloor}^{\left\lfloor x_{j+1}-1\right\rfloor} \frac{\theta_{k}}{k} t^{k}\right)\right],
$$

using the convention $x_{\ell+1}:=\infty$ and $\left\langle\boldsymbol{s}, \boldsymbol{w}_{n}(\boldsymbol{x})\right\rangle$ the standard scalar product of $\boldsymbol{w}_{n}(\boldsymbol{x})$ and $\boldsymbol{s}$. 
The first step is again to apply Cauchy's integral formula to 4.30 and to replace for all $j$ with $1 \leq j \leq \ell$ the points $x_{j}$ by $x_{j} n^{*}$ and all $s_{j}$ by $s_{j}^{*}:=s_{j} r^{-k / 2}$. Further, we use the same curve as in the proof Theorem 4.1, but with a slightly different $r^{\prime}$. Explicitly, we replace $r^{\prime}$ by

$$
r_{\ell}^{\prime}=r+v_{\ell} \text { with } v_{\ell}:=\frac{\sum_{j=1}^{\ell}\left(e^{-s_{j}^{*}}-1\right) r^{k}\left(e^{-x_{j}}-e^{-x_{j+1}}\right)}{n e^{-r}}
$$

and use the same $\delta=\left(n e^{-r}\right)^{-5 / 12}$. We then proceed to apply the saddle point method so that we arrive at

$$
\mathbb{E}_{\Theta}\left[\exp \left(-\left\langle\mathbf{s}, \widetilde{\mathbf{w}}_{n}(\mathbf{x})\right\rangle\right)\right]=\frac{\exp \left(-v_{\ell}+f_{\ell}(0)\right)}{e^{r} \sqrt{2 \pi\left(P^{\prime \prime}(r)+n e^{-r}\right)}}\left(1+O\left(r^{-1}\right)\right)
$$

with

$$
f_{\ell}(\varphi):=P\left(r^{\prime}-i \varphi\right)+n e^{-r^{\prime}} e^{i \varphi}+\sum_{j=1}^{\ell}\left(e^{-s_{j}^{*}}-1\right) \sum_{k=\left\lfloor x_{j} n^{*}\right\rfloor}^{\left\lfloor x_{j+1} n^{*}-1\right\rfloor} \frac{\theta_{m}}{m} e^{-m e^{-r^{\prime}}} e^{i \varphi}
$$

To prove the theorem, we have only to determine the coefficients of $s_{j}^{2}$ and $s_{i} s_{j}$ in $f_{\ell}(0)$. To do this, we first look at $v_{\ell}$. We use $n e^{-r}=P^{\prime}(r)$ and obtain

$$
v_{\ell}=\sum_{j=1}^{\ell}\left(e^{-x_{j}}-e^{-x_{j+1}}\right)\left(-\frac{s_{j}}{r^{k / 2}}+\frac{s_{j}^{2}}{2 r^{k}}+O\left(s_{j}^{3} r^{-3 k / 2}\right)\right)(1+O(1 / r)) .
$$

Using the expansion

$$
P\left(r_{\ell}^{\prime}\right)=P\left(r+v_{\ell}\right)=P(r)+P^{\prime}(r) v_{\ell}+\frac{1}{2} P^{\prime \prime}(r) v_{\ell}^{2}+O\left(v_{\ell}^{3} \log ^{k-2} n\right),
$$

and $P^{\prime}(r) \sim r^{k}$ and $P^{\prime \prime}(r)=O\left(r^{k-1}\right)$, we immediately get

$$
\begin{aligned}
\left.s_{j}^{2}\right]\left[P\left(r_{\ell}^{\prime}\right)\right] & =\frac{\left(e^{-x_{j}}-e^{-x_{j+1}}\right)}{2}(1+O(1 / r)) & & \text { for } 1 \leq j \leq \ell, \\
{\left[s_{i} s_{j}\right]\left[P\left(r_{\ell}^{\prime}\right)\right] } & =O(1 / r)) & & \text { for } i \neq j .
\end{aligned}
$$

Furthermore, using $n e^{-r_{\ell}^{\prime}}=n e^{-r} e^{-v_{\ell}}=P^{\prime}(r)\left(1-v_{\ell}+v_{\ell}^{2} / 2+O\left(v_{\ell}^{3}\right)\right)$, we obtain

$$
\begin{array}{rlrl}
{\left[s_{j}^{2}\right]\left[n e^{r_{\ell}^{\prime}}\right]} & =\frac{\left(e^{-x_{j}}-e^{-x_{j+1}}\right)^{2}-\left(e^{-x_{j}}-e^{-x_{j+1}}\right)}{2}(1+O(1 / r)) & & \text { for } 1 \leq j \leq \ell, \\
{\left[s_{i} s_{j}\right]\left[n e^{r_{\ell}^{\prime}}\right]} & =\left(e^{-x_{j}}-e^{-x_{j+1}}\right)\left(e^{-x_{i}}-e^{-x_{i+1}}\right)(1+O(1 / r)) & \text { for } i \neq j .
\end{array}
$$

Finally, applying Lemma 4.6, we get

$$
\sum_{j=1}^{\ell}\left(e^{-s_{j}^{*}}-1\right) \sum_{k=\left\lfloor x_{j} n^{*}\right\rfloor}^{\left\lfloor x_{j+1} n^{*}-1\right\rfloor} \frac{\theta_{m}}{m} e^{-m e^{-r^{\prime}}}=\sum_{j=1}^{\ell}\left(e^{-s_{j}^{*}}-1\right)\left(r^{k} \int_{x_{j}}^{x_{j+1}} u^{-1} e^{-u} d u+O\left(r^{k-1}\right)\right) .
$$


This implies

$$
\begin{aligned}
& {\left[s_{j}^{2}\right]\left[\frac{1}{2} \sum_{j=1}^{\ell}\left(e^{-s_{j}^{*}}-1\right) \sum_{k=\left\lfloor x_{j} n^{*}\right\rfloor}^{\left\lfloor x_{j+1} n^{*}-1\right\rfloor} \frac{\theta_{m}}{m} e^{-m e^{-r^{\prime}}}\right]=\int_{x_{j}}^{x_{j+1}} u^{-1} e^{-u} d u(1+O(1 / r)),} \\
& {\left[s_{i} s_{j}\right]\left[\sum_{j=1}^{\ell}\left(e^{-s_{j}^{*}}-1\right) \sum_{k=\left\lfloor x_{j} n^{*}\right\rfloor}^{\left\lfloor x_{j+1} n^{*}-1\right\rfloor} \frac{\theta_{m}}{m} e^{-m e^{-r^{\prime}}}\right]=O(1 / r) .}
\end{aligned}
$$

Combining all these equations, we obtain

$$
\begin{aligned}
{\left[s_{j}^{2}\right]\left[f_{\ell}(0)\right] } & =\frac{\left(e^{-x_{j}}-e^{-x_{j+1}}\right)^{2}+\int_{x_{j}}^{x_{j+1}} u^{-1} e^{-u} d u(1+O(1 / r))}{2}(1+O(1 / r)), \\
{\left[s_{i} s_{j}\right]\left[f_{\ell}(0)\right] } & =\left(e^{-x_{j}}-e^{-x_{j+1}}\right)\left(e^{-x_{i}}-e^{-x_{i+1}}\right)(1+O(1 / r)) .
\end{aligned}
$$

This completes the proof Theorem 4.3 .

4.3. Proof of Theorem 4.4. We use a similar method of proof as in [8, Section 4.3] and as in [15]. Theorem 4.3 gives us the convergence of the finite dimensional distributions. It thus remains to prove the tightness of the process. This means we have to show that the moment condition in [5, p.128] is fulfilled. We begin with the generating function. We have

Lemma 4.8 ([8, Lemma 4.10]). For $0 \leq x_{1}<x \leq x_{2}$ arbitrary and $x^{*}:=x n^{*}, x_{1}^{*}:=x_{1} n^{*}$ and $x_{2}^{*}:=x_{2} n^{*}$

$$
\begin{aligned}
& r^{2 k} \cdot h_{n} \mathbb{E}_{\Theta}\left[\left(\widetilde{w}_{n}\left(x^{*}\right)-\widetilde{w}_{n}\left(x_{1}^{*}\right)\right)^{2}\left(\widetilde{w}_{n}\left(x_{2}^{*}\right)-\widetilde{w}_{n}\left(x^{*}\right)\right)^{2}\right] \\
& =\left[t^{n}\right]\left[\left(\left(g_{x_{1}^{*}}^{x^{*}}(t)-E_{x_{1}}^{x}\right)^{2}+g_{x_{1}^{*}}^{x^{*}}(t)\right)\left(\left(g_{x^{*}}^{x_{2}^{*}}(t)-E_{x}^{x_{2}}\right)^{2}+g_{x^{*}}^{x_{2}^{*}}(t)\right) \exp \left(g_{\Theta}(t)\right)\right]
\end{aligned}
$$

with

$$
g_{a}^{b}(z):=\sum_{a \leq j<b} \frac{\vartheta_{j}}{j} z^{j} \text { and } E_{a}^{b}=\mathbb{E}_{\Theta}\left[w_{n}\left(b n^{*}\right)-w_{n}\left(a n^{*}\right)\right] \text { for } a<b
$$

We can now prove the tightness of the process $\widetilde{w}_{n}\left(x^{*}\right)$.

Lemma 4.9. We have for $0 \leq x_{1}<x \leq x_{2}<K$ with $K$ arbitrary

$$
\mathbb{E}_{\Theta}\left[\left(\widetilde{w}_{n}\left(x^{*}\right)-\widetilde{w}_{n}\left(x_{1}^{*}\right)\right)^{2}\left(\widetilde{w}_{n}\left(x_{2}^{*}\right)-\widetilde{w}_{n}\left(x^{*}\right)\right)^{2}\right]=O\left(\left(x_{2}-x_{1}\right)^{2}\right) .
$$

Proof. We use Lemma 4.8 and apply the proof of Theorem 2.5 to the function

$$
g_{n}(t):=\exp \left(g_{\Theta}(t)+\log \left(\left(g_{x_{1}^{*}}^{x^{*}}(t)-E_{x_{1}}^{x}\right)^{2}+g_{x_{1}^{*}}^{x^{*}}(t)\right)+\log \left(\left(g_{x^{*}}^{x^{*}}(t)-E_{x_{1}}^{x}\right)^{2}+g_{x^{*}}^{x_{2}^{*}}(t)\right)\right) .
$$

We claim that we can use the same curve and the same $r$ and $\delta$ as in the proof of Theorem 2.5 Theorem 4.1 and Lemma 4.6 imply immediately that $E_{x_{1}}^{x}=O\left(r^{k}\right)$ and $g_{x_{1}^{*}}^{x^{*}}\left(e^{e^{-r} e^{i \varphi}}\right)=O\left(r^{k}\right)$. It is thus immediate to show that we indeed can use the same curve and the same $r$ and $\delta$. We thus arrive at

$$
\begin{aligned}
& r^{2 k} \mathbb{E}_{\Theta}\left[\left(\widetilde{w}_{n}\left(x^{*}\right)-\widetilde{w}_{n}\left(x_{1}^{*}\right)\right)^{2}\left(\widetilde{w}_{n}\left(x_{2}^{*}\right)-\widetilde{w}_{n}\left(x^{*}\right)\right)^{2}\right] \\
& =\left(\left(g_{x_{1}^{*}}^{x^{*}}\left(e^{-e^{-r}}\right)-E_{x_{1}}^{x}\right)^{2}+g_{x_{1}^{*}}^{x^{*}}\left(e^{-e^{-r}}\right)\right)\left(\left(g_{x^{*}}^{x_{*}^{*}}\left(e^{-e^{-r}}\right)-E_{x}^{x_{2}}\right)^{2}+g_{x^{*}}^{x_{2}^{*}}\left(e^{-e^{-r}}\right)\right)(1+o(1)) .
\end{aligned}
$$


Differentiating (4.30) with respect to $s_{1}$ and substituting $s_{1}=0$ shows that

$$
E_{x_{1}}^{x}=\mathbb{E}_{\Theta}\left[\widetilde{w}_{n}\left(x^{*}\right)-\widetilde{w}_{n}\left(x_{1}^{*}\right)\right]=\frac{1}{h_{n}}\left[t^{n}\right]\left[g_{x^{*}}^{x_{2}^{*}}(t) \exp \left(g_{\Theta}(t)\right)\right]=g_{x^{*}}^{x^{*}}\left(e^{-e^{-r}}\right)(1+o(1)) .
$$

It is then clear that $g_{x_{1}^{*}}^{x^{*}}\left(e^{-e^{-r}}\right)-E_{x_{1}}^{x}=o\left(x-x_{1}\right)$. Therefore

$$
\left(\left(g_{x_{1}^{*}}^{x^{*}}\left(e^{-e^{-r}}\right)-E_{x_{1}}^{x}\right)^{2}+g_{x_{1}^{*}}^{x^{*}}\left(e^{-e^{-r}}\right)\right) r^{-k}=O\left(g_{x_{1}^{*}}^{x^{*}}\left(e^{-e^{-r}}\right) r^{-k}\right) .
$$

Applying Lemma 4.6 then shows $g_{x_{1}^{*}}^{x^{*}}\left(e^{-e^{-r}}\right) r^{-k}=O\left(x-x_{1}\right)$. Similar considerations apply for $x_{2}$. This completes the proof.

\section{TOTAL VARIATION DISTANCE}

We have proven in Section 3.2 that for each fix $b \in \mathbb{N}$

$$
\left(C_{1}, C_{2}, \ldots, C_{b}\right) \stackrel{d}{\longrightarrow}\left(Y_{1}, Y_{2}, \ldots, Y_{b}\right), \quad \text { as } n \rightarrow \infty .
$$

where $\left(C_{m}\right)_{m=1}^{b}$ denote the cycle counts on $\mathfrak{S}_{n}$ up to length $b$ and $\left(Y_{m}\right)_{m=1}^{b}$ are independent Poisson random variables with $\mathbb{E}\left[Y_{m}\right]=\frac{\theta_{m}}{m}$. Unfortunately, the convergence in (5.1) is often not strong enough, since many interesting random variables involve all or almost all cycle counts $C_{m}$. Thus, one needs estimates where $b$ and $n$ grow simultaneously. The quality of the approximation can conveniently be described in terms of the total variation distance. For all $1 \leq b \leq n$ denote by $d_{b}(n)$ the total variation distance

$$
d_{b}(n):=d_{\mathrm{TV}}\left(\mathcal{L}\left(C_{1}, C_{2}, \ldots, C_{b}\right), \mathcal{L}\left(Y_{1}, Y_{2}, \ldots, Y_{b}\right)\right)
$$

The main result of this section is

Theorem 5.1. Let $(b(n))_{n \in \mathbb{N}}$ be a sequence so that $b(n)=o\left(n^{c}\right)$ with $0<c<(3 k+3)^{-\frac{1}{k+1}}$. Then one has that

$$
d_{b}(n)=o(1)
$$

The computations in the proof of Theorem 5.1 and the similarities with the cases $\theta_{m} \approx \vartheta$ and $\theta_{m} \sim m^{\gamma}$ strongly suggest that Theorem 5.1 might not be optimal. We expect that $d_{b}(n)=o(1)$ if and only if $b(n)=o\left(n / \log ^{k}(n)\right)$. However, our current estimates for the error terms are too weak to prove this and a more sophisticated bound would be needed.

5.1. Proof of Theorem 5.1. The starting point for computing a total variation distance in a random permutation model is typically the so called conditioning relation. This has been used in [2] of the uniform measure and also in [22] for the case $\theta_{m} \sim m^{\alpha}$ and in [3] for random permutations without macroscopic cycles. Before, we can state this conditioning relation (and prove Theorem 5.1, we have to introduce some notations. We define

$$
\boldsymbol{Y}_{b}:=\left(Y_{1}, Y_{2}, \ldots, Y_{b(n)}\right) \text { and } \boldsymbol{C}_{b}:=\left(C_{1}, C_{2}, \ldots, C_{b(n)}\right)
$$

where $\left(Y_{m}\right)_{m \in \mathbb{N}}$ and $\left(C_{m}\right)_{m \in \mathbb{N}}$ are as above. Inserting the definition of the total variation distance in (5.2), we get

$$
d_{b}(n)=\frac{1}{2} \sum_{\boldsymbol{a} \in \mathbb{N}^{b}(n)}\left|\mathbb{P}_{\Theta}\left[\boldsymbol{C}_{b}=\boldsymbol{a}\right]-\mathbb{P}\left[\boldsymbol{Y}_{b}=\boldsymbol{a}\right]\right|
$$


Furthermore, we define

$$
T_{b_{1} b_{2}}:=\sum_{k=b_{1}+1}^{b_{2}} k Y_{k} \quad \text { for } b_{1}, b_{2} \in \mathbb{N} \text { with } b_{1} \leq b_{2} .
$$

The conditioning relation [1, Equation (1.15)] now states that

$$
\mathbb{P}_{\Theta}\left[\boldsymbol{C}_{b}=\boldsymbol{a}\right]=\mathbb{P}\left[\boldsymbol{Y}_{b}=\boldsymbol{a} \mid T_{0 n}=n\right] .
$$

It is straight forward to see that $(5.7)$ indeed holds also for all probability measures $\mathbb{P}_{\Theta}$. Inserting (5.7) in (5.5) and using the same computation as in the proof of [1, Lemma 3.1], one immediately obtains

$$
d_{b}(n)=\sum_{\ell=0}^{\infty} \mathbb{P}\left[T_{0 b(n)}=\ell\right]\left(1-\frac{\mathbb{P}\left[T_{b(n) n}=n-\ell\right]}{\mathbb{P}\left[T_{0 n}=n\right]}\right)_{+}
$$

with $(x)_{+}=\max \{x, 0\}$. We immediately get that

$$
d_{b}(n) \leq \mathbb{P}\left[T_{0 b(n)} \notin J\right]+\max _{\ell \in J}\left(1-\frac{\mathbb{P}\left[T_{b(n) n}=n-\ell\right]}{\mathbb{P}\left[T_{0 n}=n\right]}\right)_{+},
$$

where $J$ is an arbitrary subset of $\mathbb{N}$. We now let $J$ be the interval

$$
J:=\left[\mathbb{E}\left[T_{0 b(n)}\right]-g(n) \sqrt{\operatorname{Var}\left(T_{0 b(n)}\right)}, \mathbb{E}\left[T_{0 b(n)}\right]+g(n) \sqrt{\operatorname{Var}\left(T_{0 b(n)}\right)}\right]
$$

for some $g(n)$ with $g(n) \rightarrow \infty$ and $g(n)=o\left(\log ^{k / 2}(b(n))\right)$. Chebyshev's inequality gives

$$
d_{b}(n) \leq \max _{\ell \in J}\left(1-\frac{\mathbb{P}\left[T_{b(n) n}=n-\ell\right]}{\mathbb{P}\left[T_{0 n}=n\right]}\right)_{+}+O\left(g^{-2}(n)\right) .
$$

Thus Theorem 5.1 is proven if we can show that $\frac{\mathbb{P}\left[T_{b(n) n}=n-\ell\right]}{\mathbb{P}\left[T_{0 n}=n\right]}=1+o(1)$. The random variables $Y_{m}$ are independent Poisson distributed and thus (5.6) implies that the probability generating function of $T_{b_{1} b_{2}}$ is given by

$$
\mathbb{E}\left[z^{T_{b_{1} b_{2}}}\right]=\exp \left(\sum_{m=b_{1}+1}^{b_{2}} \frac{\theta_{m}}{m}\left(z^{m}-1\right)\right) .
$$

Using this observation and Corollary 2.3, we immediately get

$$
\frac{\mathbb{P}\left[T_{b(n) n}=n-\ell\right]}{\mathbb{P}\left[T_{0 n}=n\right]}=\frac{\exp \left(\sum_{m=1}^{b(n)} \frac{\theta_{m}}{m}\right)}{h_{n}}\left[z^{n-\ell}\right]\left[\exp \left(g_{\Theta}(z)-\sum_{m=1}^{b(n)} \frac{\theta_{m}}{m} z^{m}\right)\right] .
$$

Theorem 2.5 gives us the asymptotic behaviour of $h_{n}$. Thus it remains to compute

$$
I_{n}^{t v}:=\left[z^{n-\ell}\right]\left[\exp \left(g_{\Theta}(z)-\sum_{m=1}^{b(n)} \frac{\theta_{m}}{m} z^{m}\right)\right] .
$$

We accomplish this in similar way as in the proof of Theorem 2.5. Cauchy's integral formula and the change of variable $z=e^{-w}$ gives us

$$
I_{n}^{t v}=\frac{1}{2 \pi i} \int_{\gamma^{\prime \prime}} \exp \left(g_{\Theta}\left(e^{-w}\right)+(n-\ell) w-\sum_{m=1}^{b(n)} \frac{\theta_{m}}{m} e^{-m w}\right) d w,
$$


where $\gamma^{\prime \prime}$ is the same contour as in the proof of Theorem 2.5, see Figure 1 .

We now split the curve $\gamma^{\prime \prime}$ into two parts. Explicitly, we denote by $\gamma^{\prime \prime 2}$ the part of $\gamma^{\prime \prime}$ consisting of all $w$ with $w$ with $|w| \leq \frac{1}{b(n) \log ^{2 k}(b(n))}$ and by $\gamma^{\prime \prime 1,3}$ the remaining parts of $\gamma^{\prime \prime}$.

We begin by computing the integral over $\gamma^{\prime \prime 2}$. We note that $\gamma^{\prime \prime 2}$ includes $\gamma_{2}^{\prime \prime}$ as $e^{-r} \sim \frac{\log ^{k}(n)}{n}$ and $b(n)=O\left(n^{c}\right)$ with $c<1$. Thus $\gamma^{\prime \prime 2}$ contains the part giving the main contribution to the integral. We first show that $w \ell=o(1)$. It follows from (2.18) that $\theta_{m} \sim \log ^{k}(m)$. Since the $Y_{m}$ are independent Poisson random variables with parameter $\frac{\theta_{m}}{m}$, we get with (5.6)

$$
\mathbb{E}\left[T_{0 b(n)}\right]=\sum_{m=1}^{b(n)} m \mathbb{E}\left[Y_{m}\right]=\sum_{m=1}^{b(n)} \theta_{m} \sim b(n) \log ^{k}(b(n)) .
$$

Similarly, we obtain $\operatorname{Var}\left(T_{0 b(n)}\right) \sim b^{2}(n) \log ^{k} b(n)$. Thus we get that $\ell \sim b(n) \log ^{k}(b(n)$ and therefore $w \ell=o(1)$. Further, using $|w| \leq \frac{1}{b(n) \log ^{2 k}(b(n))}$, we get for all $w$ in $\gamma^{\prime \prime 2}$

$$
\sum_{m=1}^{b(n)} \frac{\theta_{m}}{m} \exp (-m w)=\sum_{m=1}^{b(n)} \frac{\theta_{m}}{m}(1+O(m w))=\sum_{m=1}^{b(n)} \frac{\theta_{m}}{m}+o(1) .
$$

Inserting this into the integral over $\gamma^{\prime \prime 2}$ in (5.15), we obtain

$$
I_{n}^{2}=\frac{\exp \left(-\sum_{m=1}^{b(n)} \frac{\theta_{m}}{m}+o(1)\right)}{2 \pi i} \int_{\gamma^{\prime \prime 2}} \exp \left(g_{\Theta}\left(e^{-w}\right)+n w\right) d w .
$$

The last integral is the same integral as in the proof of Theorem 2.5 . Thus we get

$$
I_{n}^{2}=\exp \left(-\sum_{m=1}^{b(n)} \frac{\theta_{m}}{m}\right) \cdot h_{n}\left(1+O\left(\log ^{-k / 2}(n)\right)\right) .
$$

Inserting (5.18) into 5.13, we obtain

$$
\frac{\mathbb{P}\left[T_{b(n) n}=n-\ell\right]}{\mathbb{P}\left[T_{0 n}=n\right]}=1+o(1)+\frac{\exp \left(\sum_{m=1}^{b(n)} \frac{\theta_{m}}{m}\right)}{h_{n}} I_{n}^{1,3},
$$

where $I_{n}^{1,3}$ is the integral over $\gamma^{\prime 11,3}$. Clearly, $\gamma^{\prime 1,3}$ is a part of $\gamma_{1}^{\prime \prime}$ and $\gamma_{3}^{\prime \prime}$. Thus we obtain as in the proof of Theorem 2.5, that

$$
\begin{aligned}
\left|I_{n}^{1,3}\right| & \leq \frac{2}{2 \pi} \int_{d(n)}^{\pi} \exp \left(\operatorname{Re}\left(g_{\Theta}\left(e^{-e^{i x}}\right)\right)-\operatorname{Re}\left(\sum_{m=1}^{b(n)} \frac{\theta_{m}}{m} e^{-m i x}\right)\right) d x \\
& \leq \frac{2 \exp \left(\sum_{m=1}^{b(n)} \frac{\theta_{m}}{m}\right)}{2 \pi} \int_{d(n)}^{\pi} \exp \left(\operatorname{Re}\left(g_{\Theta}\left(e^{-e^{i x}}\right)\right)\right) d x,
\end{aligned}
$$

with $d(n):=\frac{1}{b(n) \log ^{2 k}(b(n))}$. Using the estimate in 2.39 for $g_{\Theta}\left(e^{-e^{i x}}\right)$, we get

$$
\frac{1}{2 \pi} \int_{d(n)}^{\pi} \exp \left(\operatorname{Re}\left(g_{\Theta}\left(e^{-e^{i x}}\right)\right)\right) d x \leq \frac{1}{2 \pi} \int_{-\log (\pi)}^{e(n)} \exp \left(P(y)-\frac{9}{8} v P^{\prime \prime}(y)\right) e^{-y} d y,
$$


where $e(n):=(1+\epsilon) \log (b(n))$ with $\epsilon>0$ arbitrary. Applying Lemma 2.6 with $Q(y)=$ $P(y)-\frac{9}{8} v P^{\prime \prime}(y)-y$, we get that the last integral is bounded by

$$
\frac{1}{2 \pi} \frac{\exp \left(P(e(n))-\frac{9}{8} P^{\prime \prime}(e(n))-e(n)\right)}{P^{\prime}(e(n))-\frac{9}{8} P^{\prime \prime \prime}(e(n))} \leq \frac{2}{2 \pi} \frac{\exp (P(e(n)))}{P^{\prime}(e(n))}
$$

We now insert this inequality into (5.19) and obtain

$$
\frac{\mathbb{P}\left[T_{b(n) n}=n-\ell\right]}{\mathbb{P}\left[T_{0 n}=n\right]}=1+o(1)+O\left(\frac{\exp \left(2 \sum_{m=1}^{b(n)} \frac{\theta_{m}}{m}\right)}{h_{n}} \frac{2}{2 \pi} \frac{\exp (P(e(n)))}{P^{\prime}(e(n))}\right),
$$

Furthermore, we have by assumption $b(n)=O\left(n^{c}\right)$. This implies

$$
\sum_{m=1}^{b(n)} \frac{\theta_{m}}{m}=\log ^{k+1} b(n)+O\left(\log ^{k} b(n)\right)=c^{k+1} \log ^{k+1} n+O\left(\log ^{k}(n)\right) .
$$

Similarly, we get

$$
P(e(n))=\frac{1}{k+1}(c(1+\epsilon))^{k+1} \log ^{k+1}(n)+O\left(\log ^{k}(n)\right)
$$

Since $c<(3 k+3)^{-\frac{1}{k+1}}$ and $P(r)=\frac{1}{k+1} r^{k+1}+O\left(r^{k}\right)$, we get for $\epsilon$ small enough

$$
\begin{aligned}
\exp \left(2 \sum_{m=1}^{b(n)} \frac{\theta_{m}}{m}\right) \frac{2}{2 \pi} \frac{\exp (P(e(n)))}{P^{\prime}(e(n))} & \leq \exp \left(\frac{2 k+3}{k+1} c^{k+1}(1+\epsilon)^{k+1} \log ^{k+1}(n)\right) \\
& \leq \exp \left(\left(1-\epsilon^{\prime}\right) P(r)\right)
\end{aligned}
$$

for some $\epsilon^{\prime}>0$. It follows immediately from Theorem 2.5 that $\exp \left(\left(1-\epsilon^{\prime}\right) P(r)\right)=o\left(h_{n}\right)$. Inserting this into (5.19) completes the proof of Theorem 5.1.

\section{REFERENCES}

[1] R. Arratia, A. Barbour, and S. Tavaré. Logarithmic combinatorial structures: a probabilistic approach. EMS Monographs in Mathematics. European Mathematical Society (EMS), Zürich, 2003.

[2] R. Arratia and S. Tavaré. The cycle structure of random permutations. Ann. Probab., 20(3):1567-1591, 1992.

[3] V. Betz, H. Schäfer, and D. Zeindler. Random permutations without macroscopic cycles. Dec. 2017.

[4] V. Betz, D. Ueltschi, and Y. Velenik. Random permutations with cycle weights. Ann. Appl. Probab., 21(1):312-331, 2011.

[5] P. Billingsley. Convergence of probability measures. Wiley Series in Probability and Statistics: Probability and Statistics. John Wiley \& Sons Inc., New York, second edition, 1999. A Wiley-Interscience Publication.

[6] L. V. Bogachev and D. Zeindler. Asymptotic statistics of cycles in surrogate-spatial permutations. Communications in Mathematical Physics, pages 1-78, 2014.

[7] P. Chareka. A finite-interval uniqueness theorem for bilateral Laplace transforms. Int. J. Math. Math. Sci., pages Art. ID 60916, 6, 2007.

[8] A. Cipriani and D. Zeindler. The limit shape of random permutations with polynomially growing cycle weights. ALEA Lat. Am. J. Probab. Math. Stat., 12(2):971-999, 2015.

[9] S. Dereich and P. Mörters. Cycle length distributions in random permutations with diverging cycle weights. Random Structures Algorithms, 46(4):635-650, 2015.

[10] A. Dunn and N. Robles. Polynomial partition asymptotics. Journal of Mathematical Analysis and Applications, 459:359-384, 2018.

[11] N. M. Ercolani and D. Ueltschi. Cycle structure of random permutations with cycle weights. Random Structures Algorithms, 44(1):109-133, 2014. 
[12] W. J. Ewens. The sampling theory of selectively neutral alleles. Theoret. Population Biology, 3:87-112; erratum, ibid. 3 (1972), 240; erratum, ibid. 3 (1972), 376, 1972.

[13] P. Flajolet. Singularity analysis and asymptotics of Bernoulli sums. Theoret. Comput. Sci., 215(1-2):371-381, 1999.

[14] P. Flajolet and R. Sedgewick. Analytic Combinatorics. Cambridge University Press, New York, NY, USA, 2009.

[15] J. C. Hansen. A functional central limit theorem for the Ewens sampling formula. J. Appl. Probab., 27(1):2843, 1990.

[16] F. M. Hoppe. The sampling theory of neutral alleles and an urn model in population genetics. J. Math. Biol., 25(2):123-159, 1987.

[17] J. F. C. Kingman. The population structure associated with the Ewens sampling formula. Theoret. Population Biology, 11(2):274-283, 1977.

[18] I. G. Macdonald. Symmetric functions and Hall polynomials. Oxford Mathematical Monographs. The Clarendon Press Oxford University Press, New York, second edition, 1995. With contributions by A. Zelevinsky, Oxford Science Publications.

[19] A. Nikeghbali and D. Zeindler. The generalized weighted probability measure on the symmetric group and the asymptotic behaviour of the cycles. Annales de L'Institut Poincaré, 49, no.4:961-981, 2011.

[20] G. Pólya. Kombinatorische anzahlbestimmungen für gruppen, graphen, und chemische verbindungen. Acta Mathematica, 68:145-254, 1937.

[21] A. Shmidt and A. M. Vershik. Limit measures arising in the asymptotic theory of symmetric groups. Theory Probab. Appl., 22, No.1:70-85, 1977.

[22] J. Storm and D. Zeindler. Total variation distance and the Erdös-Turán law for random permutations with polynomially growing cycle weights. Annales de l'Institut Henri Poincar, 52(4):1614-1640, 2016.

Department of Mathematics, University of Illinois, 1409 West Green Street, Urbana, IL 61801, United States and Wolfram Research InC, 100 Trade Center Dr, Champaign, IL 61820, UNITED STATES

Email address: nirobles@illinois.edu

Email address: nicolasrewolfram.com

Department of Mathematics and Statistics, LAnCASter University, Fylde College, BailRIGG, LANCASTER LA1 4YF, UNITED KINGDOM

Email address: d.zeindlerelancaster.ac.uk 